\title{
High energy lepton colliders as the ultimate Higgs microscopes
}

\author{
Shankha Banerjee, ${ }^{a}$ Rick S. Gupta, ${ }^{b}$ Oscar Ochoa-Valeriano ${ }^{b}$ and \\ Michael Spannowsky ${ }^{b}$ \\ ${ }^{a}$ CERN, Theoretical Physics Department, \\ CH-1211 Geneva 23, Switzerland, \\ ${ }^{b}$ Institute for Particle Physics Phenomenology, Durham University, \\ South Road, Durham, DH1 3LE, U.K. \\ E-mail: shankha.banerjee@cern.ch, sandeepan.gupta@durham.ac.uk, \\ oscar.ochoa-valeriano@durham.ac.uk, michael.spannowsky@durham.ac.uk
}

ABstract: We study standard electroweak/Higgs processes at the high-energy lepton colliders ILC and CLIC. We identify a subset of three operators in the SMEFT that give leading contributions to these processes at high energies. We then perform a 'high-energy fit' including these operators. Our final bounds surpass existing LEP bounds and HL-LHC projections by orders of magnitude. Furthermore, we find that these colliders can probe scales up to tens of $\mathrm{TeV}$, corresponding to the highest scales explored in electroweak/Higgs physics.

KEywords: Beyond Standard Model, Effective Field Theories, Higgs Physics

ArXiv EPrint: 2109.14634 


\section{Contents}

1 Introduction 1

2 The leptonic high-energy primaries 2

2.1 High energy $e^{+} e^{-} \rightarrow Z h, W^{+} W^{-}$production in the D6 SMEFT 3

$2.2 Z B F$ Higgs production in the D6 SMEFT 5

3 Collider simulation $\quad 5$

3.1 Monte Carlo samples 6

$3.2 Z$ Zh channel event selection $\quad 9$

$\begin{array}{lll}3.3 & Z B F \text { channel event selection } & 10\end{array}$

4 Projected sensitivities to EFT couplings $\quad 11$

$\begin{array}{lll}5 & \text { Conclusions } & 17\end{array}$

$\begin{array}{ll}\text { A Contribution of operators to anomalous couplings } & 18\end{array}$

\section{Introduction}

The main goal of high energy physics is to probe the smallest possible length scales or equivalently the highest accessible energies. In this regard, indirect measurements can often explore much higher energies than direct measurements. A prime example is LEP, which could probe energy scales far beyond its centre-of-mass energy, i.e., up to a few $\mathrm{TeV}$, through its precise measurements at the $Z$-pole. Some of these LEP measurements provide the most powerful bounds on the scale of new physics even today. This is because indirect effects are sensitive to irrelevant operators of the Standard Model Effective Field Theory (SMEFT) (for a non-exhaustive list of SMEFT studies, see refs. [1-50]) that carry the imprint of new physics at energy scales higher than that of the studied process.

Indirect effects due to higher dimensional SMEFT operators scale either as $m_{W}^{2} / \Lambda^{2}$ or $s / \Lambda^{2}, s$ being the centre of mass energy and $\Lambda$ being the scale of new physics. As far as the latter effects are concerned, future lepton colliders such as CLIC and ILC (or in some cases even the LHC, see $[36,37,46]$ ) have a clear advantage over LEP given their higher centre-of-mass energy. Relative to low energy measurements at LEP, the same EFT effects would be enhanced by a factor of $s / s_{\text {LEP }}$ (see also refs. [51-53]). As we will show in this work, this will allow lepton colliders to probe scales up to tens of $\mathrm{TeV}$, the highest energy (and smallest length) scale probed in the electroweak/Higgs sectors.

In this work, we will identify the SMEFT operators that give the leading contributions at high energies to the standard electroweak and Higgs processes at lepton colliders 


\begin{tabular}{|c|cc|}
\hline Process & ILC $_{1000}$ & CLIC $_{3000}$ \\
\hline$e^{+} e^{-} \rightarrow Z\left(\ell^{+} \ell^{-}\right) h($ all $)$ & $\checkmark$ & $\checkmark$ \\
$e^{+} e^{-} \rightarrow e^{+} e^{-} h($ all $)$ & $\checkmark$ & $\checkmark$ \\
$e^{+} e^{-} \rightarrow W(2 j) W(2 j)$ & $\times$ & $\checkmark$ \\
$e^{+} e^{-} \rightarrow W(2 j) W\left(\ell \nu_{\ell}\right)$ & $\times$ & $\checkmark$ \\
\hline
\end{tabular}

Table 1. List of processes included in the high-energy fit performed in this work for $\mathrm{ILC}_{1000}$ and $\mathrm{CLIC}_{3000}$.

like $e^{+} e^{-} \rightarrow Z h, W^{+} W^{-}$and vector boson fusion ( $\left.V B F\right)$ production of the Higgs boson. These will be the EFT effects that are sensitive to the highest possible energy scale. We will assume that the same scale suppresses all irrelevant operators, which will allow us to perform a 'high-energy fit', taking into account only this subset of operators. We will see that only three linear combinations of operator coefficients (for a single fermion generation), the so-called 'leptonic high energy primaries', give leading contributions to all these processes at high energies. As we will see, the high energy amplitudes for these processes are closely related due to theoretical principles, namely the Goldstone Boson Equivalence theorem that relates the $e^{+} e^{-} \rightarrow Z h$ and $e^{+} e^{-} \rightarrow W^{+} W^{-}$processes and the crossing symmetry that connects the $V B F$ processes to $e^{+} e^{-} \rightarrow Z h$.

\section{The leptonic high-energy primaries}

In this section, we will identify the subset of SMEFT operators that give leading contributions to standard electroweak processes at high energy lepton colliders. We will then assume that other SMEFT operators are generated at the same scale and with Wilson coefficients not larger than those of the operators giving dominant high energy contributions. We can thus consider only this subset of operators and perform a 'high-energy leptonic fit'. We obtain projections for the case of ILC and CLIC with CoM energy $\sqrt{s}=1 \mathrm{TeV}$ and $\sqrt{s}=3 \mathrm{TeV}$, respectively. We also verify numerically that the effect of the other operators is indeed negligible. Following ref. [36], we call the linear combinations of operator coefficients that enter these high energy amplitudes as the leptonic high-energy primaries.

The processes that we include are $e^{+} e^{-} \rightarrow Z h$ and the $Z$-boson fusion production $(Z B F)$ of Higgs bosons for our ILC projections. For the CLIC projections, we also include $e^{+} e^{-} \rightarrow W^{+} W^{-}$process. While the results for the $Z h$ and $Z B F$ processes are based on our own collider analyses, we use the results of ref. [51] for the $e^{+} e^{-} \rightarrow W^{+} W^{-}$process. We summarise this list of processes including the decay channels we have considered in table 1.

A remarkable fact about the SMEFT contributions to these processes is that the same set of 3 leptonic high-energy primaries dominate the SMEFT contribution at high energies in each case. ${ }^{1}$ We thus statistically combine the results from each process to obtain the

\footnotetext{
${ }^{1}$ See, also ref. [51] where the Drell-Yan process (not included in this work) has been shown to be sensitive to very high scales. The high-energy amplitude for this process involves an independent set of operators from the ones considered here.
} 
$\mathrm{SM}$

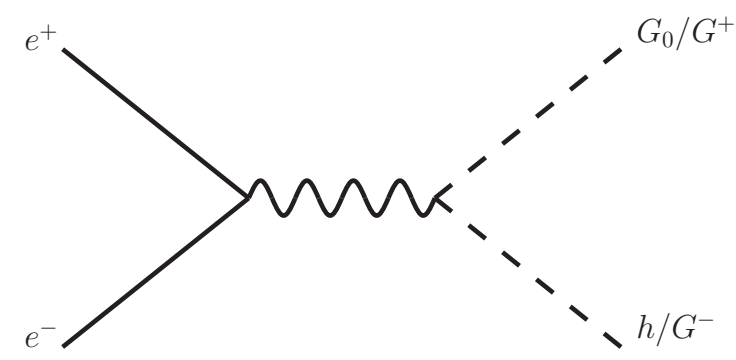

D6

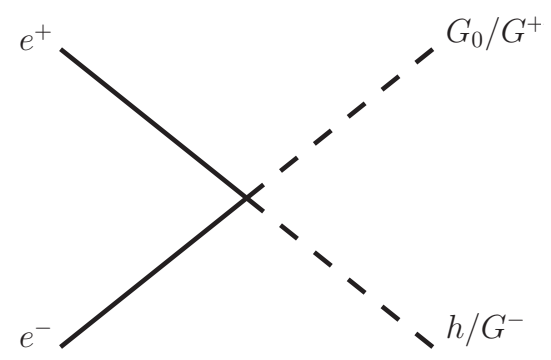

Figure 1. The leading high energy contribution to $e^{+} e^{-} \rightarrow Z h, W^{+} W^{-}$amplitudes in the SM and D6 SMEFT using the Goldstone Boson Equivalence Theorem.

best bounds on this three-dimensional space. ${ }^{2}$ We will now describe the above processes in the high-energy limit in SMEFT and discuss the theoretical principles underlying the relationship between them.

\subsection{High energy $e^{+} e^{-} \rightarrow Z h, W^{+} W^{-}$production in the D6 SMEFT}

Owing to the Goldstone Boson Equivalence Theorem [54, 55], the production of longitudinally-polarised bosons are correlated in the high-energy limit, $E \gg m_{W}$, where $E=\sqrt{s}$. The externally produced longitudinally-polarised states of the gauge bosons can be represented in the Feynman diagrams by the corresponding Goldstone bosons, up to a factor of $m_{W} / E$. To understand the high-energy behaviour of these processes, it is, therefore, sufficient to study these in the unbroken phase, where the SM symmetry of $\mathrm{SU}(2)_{L} \times \mathrm{U}(1)_{Y}$ is intact. In the unbroken phase, the electroweak bosons are massless, and the Goldstone bosons reside in the same doublet as the Higgs boson. Hence, it follows that at high energies, the processes, $e^{+} e^{-} \rightarrow W^{+} W^{-}, Z h-$ which get their dominant contribution from the longitudinal final-states, $W_{L} W_{L}, Z_{L} h-$ are correlated. This observation was made in the context of the corresponding processes for hadronic colliders in [36] (see also ref. [37]).

In the SM, as well as the D6 SMEFT, the high-energy amplitude for these processes can be computed by evaluating the first diagram in figure 1 where the dashed lines represent the respective Goldstone bosons. The leading contribution to these processes in the D6 SMEFT, shown in the second diagram in figure 1, arises from eeh $G_{0}$ and $e e G^{+} G^{-}$ contact terms, $G_{0}, G^{ \pm}$being the SM Goldstone modes. These contact terms are generated upon expanding the operators in table 2. As the EFT diagram has no $Z$-propagator, the corresponding amplitude grows quadratically with energy compared to the SM. The D6 amplitudes for these processes can thus be simply read off from the coefficient of the eeh $G_{0}$ and $e e G^{+} G^{-}$vertices generated upon expanding the operators in table 2. This gives, for

\footnotetext{
${ }^{2}$ The EFT corrections to $W$-boson fusion $(W B F)$ process $e^{+} e^{-} \rightarrow \nu_{e} \bar{\nu}_{e} h$ at high energies can also be completely described by the same three operators and this process must be included to obtain the best possible bounds. We will keep an analysis of this process for future work.
} 
$s \gg m_{Z}^{2}$,

$$
\begin{aligned}
\frac{\delta \mathcal{A}_{e_{R} e_{R} \rightarrow W W}}{\mathcal{A}_{e_{R} e_{R} \rightarrow W W}^{S M}} & =\frac{\delta \mathcal{A}_{e_{R} e_{R} \rightarrow Z h}}{\mathcal{A}_{e_{R} e_{R} \rightarrow Z h}^{S M}}=\frac{1}{2 q_{e_{R}}^{Z}} \frac{s}{m_{Z}^{2}} \alpha_{e_{R}} \\
\frac{\delta \mathcal{A}_{e_{L} e_{L} \rightarrow Z h}}{\mathcal{A}_{e_{L} e_{L} \rightarrow Z h}^{S M}} & =\frac{1}{2 q_{e_{L}}^{Z}} \frac{s}{m_{Z}^{2}}\left(\alpha_{L 1}+\alpha_{L 3}\right) \\
\frac{\delta \mathcal{A}_{e_{L} e_{L} \rightarrow W W}}{\mathcal{A}_{e_{L} e_{L} \rightarrow W W}^{S M}} & =\frac{1}{2 q_{e_{L}}^{Z}} \frac{s}{m_{Z}^{2}}\left(\alpha_{L 1}-\alpha_{L 3}\right),
\end{aligned}
$$

where $\mathcal{A}^{S M}$ and $\delta \mathcal{A}$ are the SM amplitude and the EFT contribution respectively for the given process and $q_{f}^{Z}=\left(T_{3 f}-Q_{f} s_{\theta_{W}}^{2}\right)$. Here, $\alpha_{e_{R}}, \alpha_{L 1}$ and $\alpha_{L 3}$ are the leptonic high energy primaries which are the linear combinations of Wilson coefficients of D6 SMEFT operators. The 'Strongly Interacting Light Higgs' (SILH) Lagrangian [2] is especially suited to parametrise universal new physics effects. The leptonic high energy primaries for the universal case are expressed in terms of the SILH operators as follows,

$$
\begin{aligned}
\alpha_{L 1} & =\frac{\alpha_{e_{R}}}{2}=\frac{m_{W}^{2} t_{\theta_{W}}^{2}}{\Lambda^{2}}\left(c_{B}+c_{H B}-c_{2 B}\right) \\
\alpha_{L 3} & =-\frac{m_{W}^{2}}{\Lambda^{2}}\left(c_{W}+c_{H W}-c_{2 W}\right) .
\end{aligned}
$$

We see that only two of these three directions remain independent in the universal case.

In the appendix A we show how the Wilson coefficients above can be written in terms of some universal 'pseudo-observables' that can be independently constrained in other collider processes. This allows us to write,

$$
\begin{aligned}
& \alpha_{L 1}=\frac{\alpha_{e_{R}}}{2}=-t_{\theta_{W}}^{2}\left(\delta \kappa_{\gamma}-\hat{S}-\delta g_{1}^{Z} c_{\theta_{W}}^{2}+Y\right) \\
& \alpha_{L 3}=\delta g_{1}^{Z} c_{\theta_{W}}^{2}+W .
\end{aligned}
$$

For the general case we use the Warsaw basis [3] (see second column of table 2) and the expressions for the high-energy primaries become especially simple,

$$
\alpha_{e_{R}}=-\frac{c_{R}^{e} v^{2}}{\Lambda^{2}}, \quad \alpha_{L 1}=-\frac{c_{L}^{l,(1)} v^{2}}{\Lambda^{2}} \alpha_{L 3}=-\frac{c_{L}^{l,(3)} v^{2}}{\Lambda^{2}}
$$

Again, using the expressions in eq. (A.9) in appendix A we rewrite the above equations in terms of the anomalous couplings that can be independently constrained,

$$
\begin{aligned}
& \alpha_{L 1}=\frac{c_{\theta_{W}}}{g}\left(\delta g_{e_{L}}^{Z}+\delta g_{\nu_{L}}^{Z}\right)+s_{\theta_{W}}^{2} \delta g_{1}^{Z}-t_{\theta_{W}}^{2} \delta \kappa_{\gamma} \\
& \alpha_{L 3}=\frac{c_{\theta_{W}}}{g}\left(\delta g_{e_{L}}^{Z}-\delta g_{\nu_{L}}^{Z}\right)+c_{\theta_{W}}^{2} \delta g_{1}^{Z} \\
& \alpha_{e_{R}}=\frac{2 c_{\theta_{W}}}{g} \delta g_{e_{R}}^{Z}+2 s_{\theta_{W}}^{2} \delta g_{1}^{Z}-2 t_{\theta_{W}}^{2} \delta \kappa_{\gamma} .
\end{aligned}
$$




\begin{tabular}{|l|l|}
\hline SILH Basis & Warsaw Basis \\
\hline $\mathcal{O}_{W}=\frac{i}{2}\left(H^{\dagger} \tau^{a} \stackrel{\leftrightarrow}{D^{\mu}} H\right) D^{\nu} W_{\mu \nu}^{a}$ & $\mathcal{O}_{L}^{l,(3)}=\left(\bar{L} \sigma^{a} \gamma^{\mu} L\right)\left(i H^{\dagger} \sigma^{a} \stackrel{\leftrightarrow}{D}_{\mu} H\right)$ \\
$\mathcal{O}_{B}=\left(H^{\dagger} \stackrel{\leftrightarrow}{D}^{\mu} H\right) \partial^{\nu} B_{\mu \nu}$ & $\mathcal{O}_{L}^{l,(1)}=\left(\bar{L} \gamma^{\mu} L\right)\left(i H^{\dagger} \overleftrightarrow{D}_{\mu} H\right)$ \\
$\mathcal{O}_{H W}=i g\left(D^{\mu} H\right)^{\dagger} \sigma^{a}\left(D^{\nu} H\right) W_{\mu \nu}^{a}$ & $\mathcal{O}_{R}^{e}=\left(\bar{e}_{R} \gamma^{\mu} e_{R}\right)\left(i H^{\dagger} \stackrel{\leftrightarrow}{D}_{\mu} H\right)$ \\
$\mathcal{O}_{H B}=i g^{\prime}\left(D^{\mu} H\right)^{\dagger}\left(D^{\nu} H\right) B_{\mu \nu}$ & \\
$\mathcal{O}_{2 W}=-\frac{1}{2}\left(D^{\mu} W_{\mu \nu}^{a}\right)^{2}$ & \\
$\mathcal{O}_{2 B}=-\frac{1}{2}\left(\partial^{\mu} B_{\mu \nu}\right)^{2}$ & \\
\hline
\end{tabular}

Table 2. Dimension-six operators contributing to $e^{+} e^{-} \rightarrow Z h, W^{+} W^{-}$and $Z B F$ Higgs production processes at high energies.

\section{2 $\quad Z B F$ Higgs production in the D6 SMEFT}

As far as the $Z B F$ process is concerned, the diagram corresponding to it can be obtained just by rotating the one for $e^{+} e^{-} \rightarrow Z h$ (see figure 2). In other words, the two processes are related by crossing-symmetry (see also ref. [46]), and the amplitude for the $Z B F$ process can be obtained simply by replacing $s \rightarrow t$, i.e.,

$$
\begin{aligned}
& \frac{\delta \mathcal{A}_{e_{R} Z \rightarrow e_{R} h}}{\mathcal{A}_{e_{R} Z \rightarrow e_{R} h}^{S M}}=\frac{\delta \mathcal{A}_{e_{R} e_{R} \rightarrow Z h}}{\mathcal{A}_{e_{R} e_{R} \rightarrow Z h}^{S M}}(s \rightarrow t)=\frac{t}{m_{Z}^{2}} \alpha_{e_{R}} \\
& \frac{\delta \mathcal{A}_{e_{L} Z \rightarrow e_{L} h}}{\mathcal{A}_{e_{L} Z \rightarrow e_{L} h}^{S M}}=\frac{\delta \mathcal{A}_{e_{L} e_{L} \rightarrow Z h}}{\mathcal{A}_{e_{L} e_{L} \rightarrow Z h}^{S M}}(s \rightarrow t)=\frac{t}{m_{Z}^{2}}\left(\alpha_{L 1}+\alpha_{L 3}\right),
\end{aligned}
$$

where $s$ and $t$ are the usual Mandelstam variables.

\section{Collider simulation}

We now discuss the collider analysis that helps us in extracting the signal from background processes at future linear $e^{+} e^{-}$colliders [56-58]. The single-Higgs production mechanisms we study are $Z\left(\ell^{+} \ell^{-}\right) h$, with $\ell=\{e, \mu, \tau\}$, and $Z B F$, targeting a semi-inclusive search of those decays of the Higgs boson that do not yield final-state charged leptons ${ }^{3}$ to avoid redundancies in the reconstruction of the two-lepton system. Although both processes interfere for final-state electrons, their invariant masses are in themselves powerful discriminating variables that permits us to define mutually exclusive analysis categories. While leptonic decays of the $Z$-boson are suppressed compared to the hadronic ones, we sacrifice event rates for clean final-state signatures. Hence, the signal event topologies are characterised by one pair of opposite-sign same-flavour (OSSF) leptons and additional activity in the form of jets, photons, missing energy, or a combination of the latter.

\footnotetext{
${ }^{3}$ Although it is not an inclusive search, we still keep $\sim 90 \%$ of Higgs decays.
} 


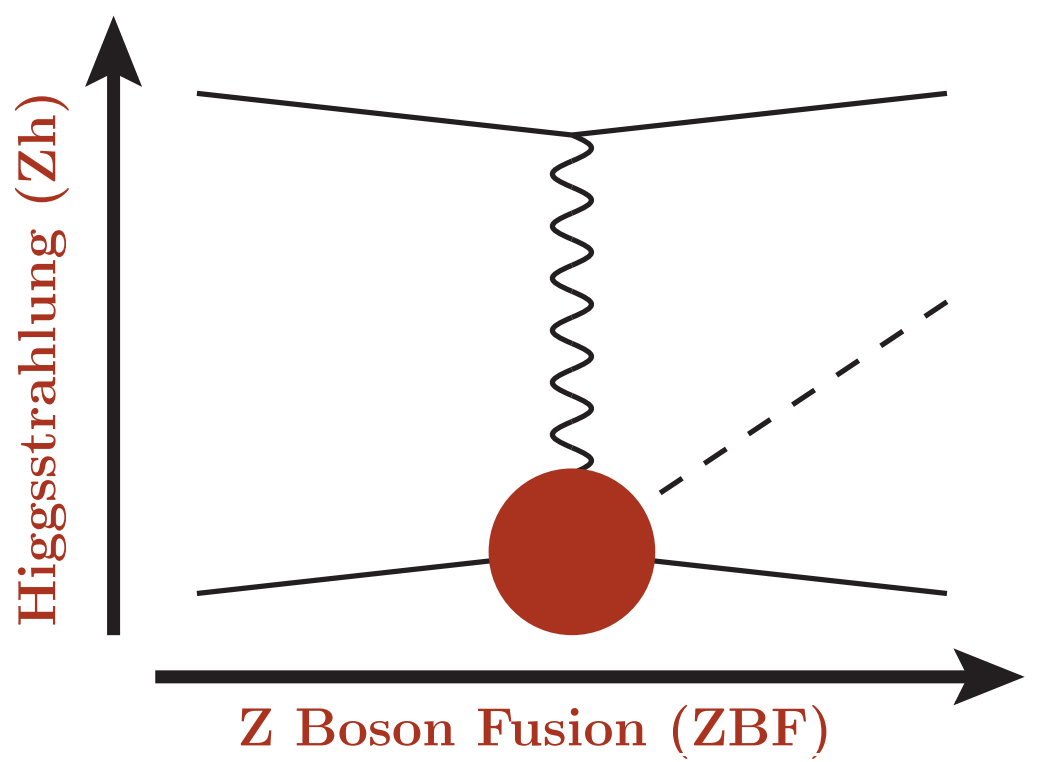

Figure 2. Crossing symmetry that relates the $Z h$ and $Z B F$ Higgs production processes. The amplitudes of these are the same up to an exchange of the Mandelstam variables $s$ and $t, s \leftrightarrow \mathrm{t}$. In consequence, the same direction in SMEFT space control $Z h$ at high $s$ and $Z B F$ at high $t$.

\subsection{Monte Carlo samples}

To obtain bounds on the relevant anomalous couplings, we implement our UFO [59] model using FeynRules [60]. We use this model to generate signal and background samples, where we include the interference and the squared terms ensuing from the D6 operators for the signal ones. We consider the EFT-driven $e^{+} e^{-} \rightarrow \ell^{+} \ell^{-} h$ and $e^{+} e^{-} \rightarrow e^{+} e^{-} h$ signal processes, where we name the former $Z h$-like and the latter $Z B F$-like. In both cases, we consider the same set of background processes, namely $2 \ell \gamma, 2 \ell 2 \gamma, 2 \ell 2 \nu_{\ell}, 2 \ell 2 \nu_{\ell} \gamma$, and $2 \ell 2 j$. At high energies, we further take into account $2 \ell 2 V(V=W, Z)$, as well as the fully-leptonic $t \bar{t}$ process. For $Z h$-like backgrounds $\ell=\{e, \mu, \tau\}$, while for the $Z B F$-like ones $\ell=\{e, \tau\} .{ }^{4}$ We neglect reducible backgrounds arising from processes where jets or photons can be misidentified as charged leptons, and consider the SM-driven signal processes as the major sources of background noise.

We consider the centre-of-mass energies $\sqrt{s}=\{250,1000,3000\} \mathrm{GeV}$, where the first and second entries correspond to the low- and high-energy phases of the International Linear Collider (ILC) [61, 62], respectively, and the third one corresponds to the highenergy run of the Compact Linear Collider (CLIC) [51, 63]. We use, from now on, the nomenclature $\operatorname{ILC}_{250,1000}\left(\mathrm{CLIC}_{3000}\right)$ to refer to the ILC (CLIC) collider run at the $\sqrt{s}$ subscript. We further follow the recommendations of ref. [64] to incorporate longitudinal beam polarisation $[65-68]^{5}$ at the considered energies and luminosities to carry out our

\footnotetext{
${ }^{4}$ Note that $\nu_{\ell}$ is always the set of all three flavours of neutrinos.

${ }^{5}$ Throughout the text, the term polarisation stands for longitudinal polarisation, and we omit longitudinal for simplicity.
} 


\begin{tabular}{|c|ccc|}
\hline Collider & $\sqrt{s}[\mathrm{GeV}]$ & $P_{e^{+}}, P_{e^{-}}[\%]$ & $\mathcal{L}\left[\mathrm{fb}^{-1}\right]$ \\
\hline $\mathrm{ILC}_{250}$ & 250 & $\pm 30, \mp 80$ & 2000 \\
$\mathrm{ILC}_{1000}$ & 1000 & $\pm 20, \mp 80$ & 8000 \\
$\mathrm{CLIC}_{3000}$ & 3000 & $0, \mp 80$ & 5000 \\
\hline
\end{tabular}

Table 3. Summary of the main configuration settings for the future linear $e^{+} e^{-}$colliders considered in our study (see text for details). The first column corresponds to the collider, the second column shows the centre-of-mass energy, $\sqrt{s}$, in $\mathrm{GeV}$, the third column corresponds to the polarisation $P_{e^{+}}$, $P_{e^{-}}$of the $e^{+} e^{-}$beams, and the last column shows the total integrated luminosity, $\mathcal{L}$, in $\mathrm{fb}^{-1}$.

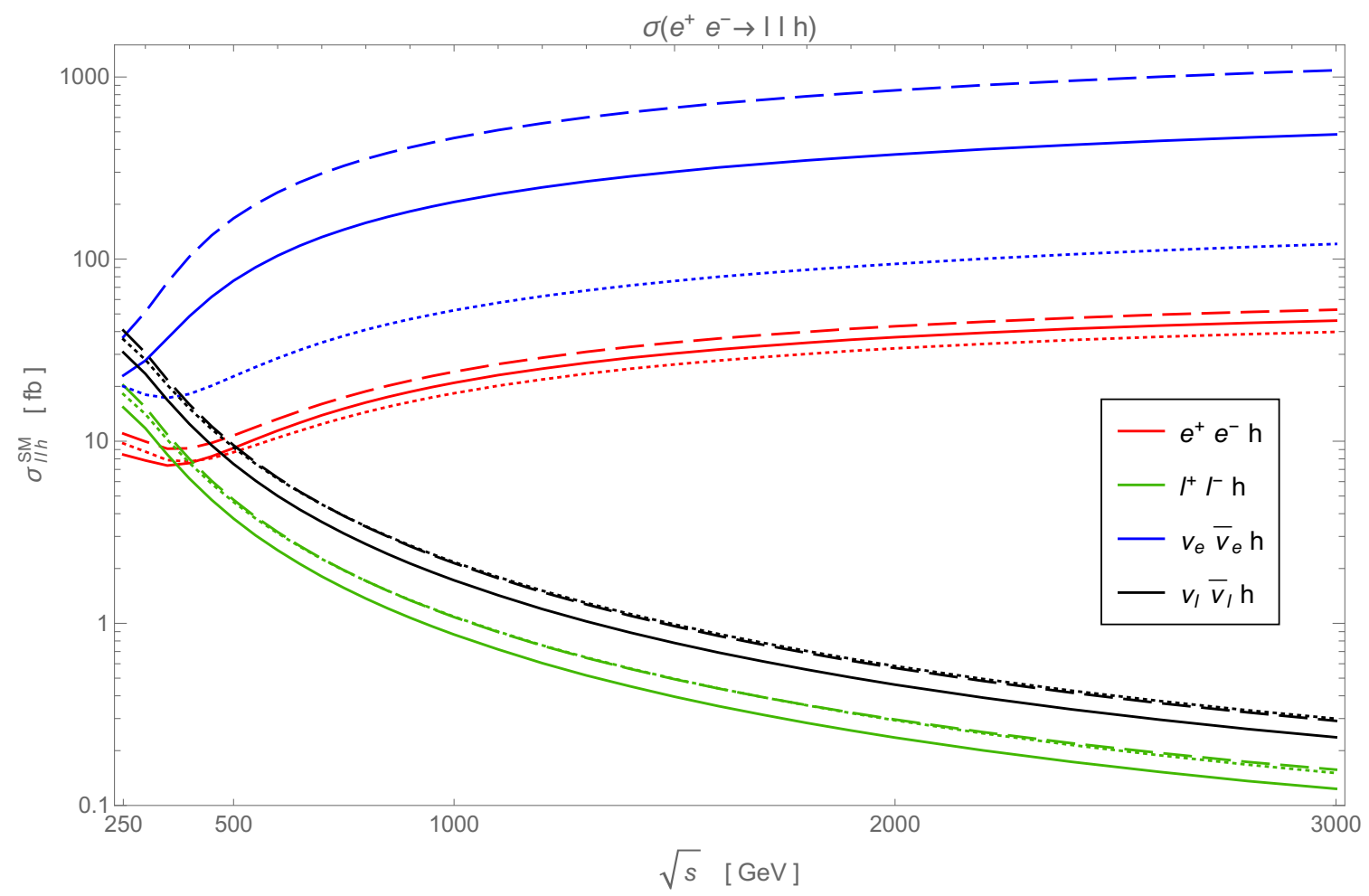

Figure 3. Interplay between beam polarisation $P_{e^{+}}, P_{e^{-}}$and cross-sections, $\sigma_{\ell \ell h}^{\mathrm{SM}}$, in fb, as functions of the centre-of-mass energy, $\sqrt{s}$, in $\mathrm{GeV}$, for single-Higgs production in association with a pair of leptons, where in the legend, $\ell=\{\mu, \tau\}$, for unpolarised beams (solid), $P_{e^{+}}, P_{e^{-}}=+50 \%,-50 \%$ (dashed), and $P_{e^{+}}, P_{e^{-}}=-50 \%,+50 \%$ (dotted). Note that both $e^{+} e^{-} h$ and $\nu_{e} \bar{\nu}_{e} h$ final states consist of $Z h-Z B F$ interference for the former, and $Z h-W B F$ for the latter. The chosen beam polarisations are meant for illustration purposes and do not necessarily correspond to the projected configuration of future colliders. 
study. Denoting $\mathcal{L}$ as the integrated luminosity in $\mathrm{fb}^{-1}$, and $P_{e^{+}}, P_{e^{-}}{ }^{6}$ as the polarisations of the positron and electron beam, respectively, the collider- and $\sqrt{s}$-dependent configurations we take into account are $\mathrm{ILC}_{250}: \mathcal{L}=2000, P_{e^{+}}, P_{e^{-}}= \pm 30 \%, \mp 80 \% ; \mathrm{ILC}_{1000}$ : $\mathcal{L}=8000, P_{e^{+}}, P_{e^{-}}= \pm 20 \%, \mp 80 \%$; and $\mathrm{CLIC}_{3000}: \mathcal{L}=5000, P_{e^{+}}, P_{e^{-}}=0 \%, \mp 80 \% .{ }^{7} \mathrm{We}$ call left and right polarisation the cases where $P_{e^{+}} \geq 0, P_{e^{-}}<0$ and $P_{e^{+}} \leq 0, P_{e^{-}}>0$, respectively. The interplay between single-Higgs production cross-section $\sigma_{\ell \ell h}^{\mathrm{SM}}$ in association with a pair of leptons and beam polarisation $P_{e^{+}}, P_{e^{-}}$in $e^{+} e^{-}$collisions as a function of the centre-of-mass energy $\sqrt{s}$ is shown in figure $3,{ }^{8}$ and a summary of the future colliders considered in this work is shown in table 3 .

To estimate the sensitivity reach of our study at the given energies and luminosities, we use MadGraph5_aMC@NLO [69] to generate the leading-order (LO) Monte Carlo event samples, and then shower/hadronise our events with Pythia 8.2 [70]. DELPHES 3 [71] is used to perform a fast detector simulation with the ILD Tune $[72,73]$ for the $\operatorname{ILC}_{250}$ and ILC $_{1000}$ scenarios, while for CLIC $_{3000}$ we use the CLICdet Stage3 Tune [74, 75]. We impose a minimal set of cuts at the generator level on the final-state objects, namely $p_{T}^{\ell, j, \gamma}>5 \mathrm{GeV}$, $m_{j j}>10 \mathrm{GeV},\left|y^{j, \gamma}\right|\left(\left|y^{\ell}\right|\right)<5(3)$, and $\Delta R_{a, b}>0.1$, where $\Delta R_{a, b}=\sqrt{(\Delta \phi)^{2}+(\Delta y)^{2}}$ is the angular distance in the $\phi-y$ plane $^{9}$ between any two final-state objects $a, b$. We further require a cut on the invariant mass $m_{\ell^{+} \ell^{-}}$of the dilepton system in order to classify our Monte Carlo samples on the mutually exclusive categories $Z h$ - and $Z B F$-like - for the $Z h$-like samples, $m_{\ell^{+} \ell^{-}} \in[70,110] \mathrm{GeV}$, whereas for the $Z B F$-like ones we impose a $\sqrt{s}$-dependent cut, $m_{\ell^{+} \ell^{-}}^{\sqrt{s}}$, as follows: $m_{\ell^{+} \ell^{-}}^{250}>100 \mathrm{GeV}, m_{\ell^{+} \ell^{-}}^{1000}>300 \mathrm{GeV}$, and $m_{\ell^{+} \ell^{-}}^{3000}>1000 \mathrm{GeV}$. An additional cut $y^{\ell^{+}} \cdot y^{\ell^{-}}<0$ is imposed on the $Z B F$-like samples to ensure that the final-state leptons lie in opposite hemispheres of the detector.

The ability of a lepton collider to exploit the four-momentum conservation given that the $p_{e^{+} e^{-}}$initial state is well known allows us to uniquely specify the decay of the Higgs boson,

$$
p_{h}=p_{e^{+} e^{-}}-p_{\ell^{+} \ell^{-}},
$$

where $p_{\ell^{+} \ell^{-}}$corresponds to the four-momentum of the final-state dilepton system. This allows us to perform model-independent studies of the Higgs width, $\Gamma_{h}$, and its inclusive production rate, among others. In practice we make use of the dimensionful variable derived from eq. (3.1) called the recoil mass, $m_{\text {recoil }}^{2}$, as a function of the centre-of-mass energy $\sqrt{s}$, the invariant mass $m_{\ell^{+} \ell^{-}}$, and the energy $E_{\ell^{+} \ell^{-}}$of the final-state dilepton system,

$$
m_{\text {recoil }}^{2} \equiv s-2 \sqrt{s} E_{\ell^{+} \ell^{-}}+m_{\ell^{+} \ell^{-}}^{2},
$$

as a discriminator against background noise. It is expected that the $m_{\text {recoil }}$ distribution shows a narrow peak centred at $m_{h}=125 \mathrm{GeV}$ given the small width $\Gamma_{h} \sim 4.088 \mathrm{MeV}$ of

\footnotetext{
${ }^{6}$ In this notation, $+100 \%$ corresponds to a fully right-handed polarised beam, and $-100 \%$ to a fully left-handed polarised beam.

${ }^{7}$ Note that at a given $\sqrt{s}$ we have two polarisation combinations, to each of which corresponds a different fraction of the integrated luminosity, i.e., two colliders in one with different luminosities each.

${ }^{8}$ We impose minimal generator-level cuts to compute these, such as $p_{T}^{\ell}>1.0 \mathrm{GeV},\left|y^{\ell}\right|<5.0$, and $\Delta R_{\ell^{+}, \ell^{-}}>0.1$.

${ }^{9}$ Although it is customary to use the polar angle $\theta$ in lepton colliders, we use instead the rapidity $y$ for consistency with the generator and detector simulator parameters. In the massless limit $\theta \equiv 2 \arctan \left(e^{-y}\right)$.
} 
the Higgs boson, in line with the LHC HXSWG Report 4 [76], provided good detector resolution for the leptons' momenta is in place. Initial- (ISR) and final-state radiation (FSR), as well as beamstrahlung, produce collinear photons that can smear the $m_{\text {recoil }}$ distribution - experimentally this effect can be controlled by imposing selection cuts on the $p_{T}$ of the outgoing dilepton system, since photons escaping the detector acceptance will not contribute to a large amount of transverse momentum of the observed objects.

\subsection{Zh channel event selection}

We perform a simplified cut-and-count analysis that allows us to suppress background processes from the SM-driven $e^{+} e^{-} \rightarrow \ell^{+} \ell^{-} h$ at $\mathrm{ILC}_{250,1000}$ and $\mathrm{CLIC}_{3000}{ }^{10}$ on the stable final-state objects after running a detector simulation (see section 3.1). The Higgs-strahlung channel is an excellent way to identify on-shell $Z$-bosons and make use of eq. (3.1) to reconstruct the Higgs boson in a "model-independent" way. Being an $s$-channel process, its cross-section decreases with the centre-of-mass energy as $\sigma \sim 1 / s$, as previously shown in figure 3. As the dominant production mode at low energies, the $\mathrm{ILC}_{250}$ is a Higgs factory that provides high statistics for the study of gauge-Higgs couplings with leptonically decaying $Z$-bosons that yield a clean signature. However, at high energies, the cross-section is suppressed with respect to the $t$-channel processes, and hadronically decaying $Z$-bosons can enhance the total cross-section since its branching ratio is the largest. Although the hadronic modes of the $Z$-boson dominate over the leptonic ones, $Z \rightarrow q \bar{q}$ decays are less clean. As we target those Higgs decays that do not yield charged final-state leptons, QCD activity associated with $Z$ decays might present challenges in reconstructing the latter, requiring detailed background analysis.

A minimal set of selection cuts is applied to the Monte Carlo samples and varies slightly depending on the $\sqrt{s}$ under consideration. We demand the presence of exactly one pair of OSSF leptons with $m_{\ell^{+} \ell^{-}} \in[86,96] \mathrm{GeV}$ - the resolution achievable at lepton colliders provides a clean reconstruction of the $Z$-boson peak $\sim 91 \mathrm{GeV}$, regardless of the collision energy. At the $\mathrm{ILC}_{250,1000}$ and $\mathrm{CLIC}_{3000}$ we require the transverse momentum of the dilepton system $p_{T}^{\ell^{+} \ell^{-}} \in\{[40,70],[400,490],[1350,1500]\} \mathrm{GeV}$, respectively. Finally, from eq. (3.2), we apply a hard cut on the recoil system's mass $m_{\text {recoil }} \in[123,127] \mathrm{GeV}$, which makes use of the fact that the $m_{\text {recoil }}$ distribution shows a narrow peak at $m_{h} \sim 125 \mathrm{GeV}$, on top of a continuum background. The $m_{\ell^{+} \ell^{-}}$and $m_{\text {recoil }}$ distributions for the $\mathrm{ILC}_{1000}$ with left polarisation can be seen in figure 4 , and the impact of the event selection on the cross-sections for the dominant SM processes under consideration is shown in table 4 for the three centreof-mass energies, as well as for the left and right polarisations. Since we are interested in contact operators that grow with energy, we find that the ILC $_{250}$ results are not sensitive to the effects of these operators. Hence, we will only focus on the high-energy regime, i.e., $\mathrm{ILC}_{1000}$ and $\mathrm{CLIC}_{3000}$. It is important to highlight that the kinematic distributions used to perform the event selection show similar shapes/behaviour in both polarisation settings.

\footnotetext{
${ }^{10}$ Similar studies focusing on inclusive or $h \rightarrow b \bar{b}$ Higgs decays can be found in, e.g., refs. [15, 77, 78].
} 


\begin{tabular}{|c|c|c|c|c|c|c|}
\hline$\sigma_{\sqrt{s}}^{\text {stage }}[\mathrm{fb}]$ & $\mathrm{SM}$ & $2 \ell \gamma$ & $2 \ell 2 \gamma$ & $2 \ell 2 \nu_{\ell}$ & $2 \ell 2 \nu_{\ell} \gamma$ & $2 \ell 2 j$ \\
\hline$\sigma_{250}^{\text {in }}$ & $29.85 / 26.26$ & $5107.28 / 4735.02$ & $316.95 / 287.56$ & $651.88 / 101.26$ & $68.75 / 8.21$ & $264.22 / 181.23$ \\
$\sigma_{250}^{\text {out }}$ & $6.99 / 6.14$ & $<10^{-6}$ & $0.40 / 0.32$ & $1.02 / 0.12$ & $0.13 / 0.04$ & $0.22 / 0.08$ \\
\hline$\sigma_{1000}^{\text {in }}$ & $1.45 / 1.45$ & $105.60 / 87.80$ & $19.46 / 16.48$ & $318.14 / 37.86$ & $39.18 / 4.91$ & $28.24 / 17.80$ \\
$\sigma_{1000}^{\text {out }}$ & $0.33 / 0.31$ & $0.017 / 0.013$ & $0.0064 / 0.0053$ & $0.0046 / 0.0024$ & $3 / 2\left(\times 10^{-4}\right)$ & $0.0140 / 0.0072$ \\
\hline$\sigma_{3000}^{\text {in }}$ & $0.17 / 0.17$ & $6.17 / 5.09$ & $1.65 / 1.37$ & $376.87 / 43.02$ & $61.51 / 7.18$ & $2.29 / 1.31$ \\
$\sigma_{3000}^{\text {out }}$ & $0.026 / 0.025$ & $12 / 9\left(\times 10^{-4}\right)$ & $9.64 / 6.09\left(\times 10^{-5}\right)$ & $3.8 / 1.9\left(\times 10^{-4}\right)$ & $61.5 / 3.6\left(\times 10^{-6}\right)$ & $8.3 / 4.9\left(\times 10^{-4}\right)$ \\
\hline
\end{tabular}

Table 4. Cross-sections, $\sigma$, in $\mathrm{fb}$, for the $Z h$-like SM-driven signal $e^{+} e^{-} \rightarrow \ell^{+} \ell^{-} h$ and its dominant backgrounds before $($ in $)$ and after (out) event selection at $\sqrt{s}=\{250,1000,3000\} \mathrm{GeV}$. We consider the left and right beam polarisations $P_{e^{+}}, P_{e^{-}}$for each $\sqrt{s}$, reported as left/right (see text for details). For the $t \bar{t}, \ell^{+} \ell^{-} W^{+} W^{-}$, and $\ell^{+} \ell^{-} Z Z$ channels, $\sigma^{\text {in }}$ (in fb) $\sim 3.47 / 0.62,4.44 / 0.41$, and $0.22 / 0.12$, respectively at $1 \mathrm{TeV}$. At $3 \mathrm{TeV}$, the respective numbers are 0.08/0.01, 1.77/0.21, and 0.06/0.03. For all cases, $\sigma^{\text {out }}<10^{-6} \mathrm{fb}$. For $\sqrt{s}=250 \mathrm{GeV}$, the kinematic phase space is not open for any of these three channels.
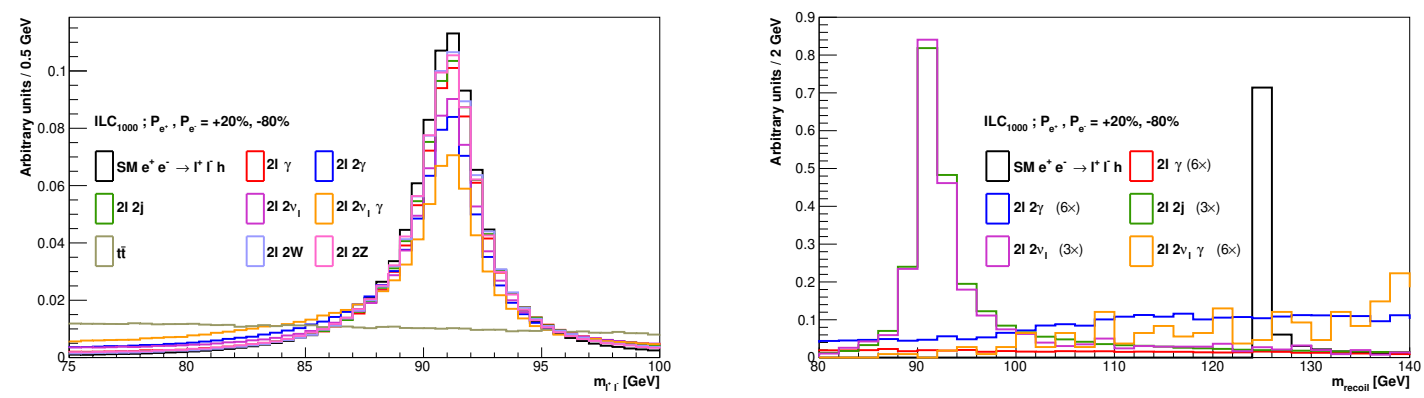

Figure 4. Some plots of the $\mathrm{ILC}_{1000} Z h$ selection with the left polarisation for the SM-driven $e^{+} e^{-} \rightarrow \ell^{+} \ell^{-} h$ (black), $2 \ell \gamma$ (red), $2 \ell 2 \gamma$ (blue), $2 \ell 2 j$ (green), $2 \ell 2 \nu_{\ell}$ (magenta), $2 \ell 2 \nu_{\ell} \gamma$ (orange), $t \bar{t}$ (brown), $2 \ell 2 W$ (lilac), and $2 \ell 2 Z$ (pink) processes. All histograms are normalised to unity. Left: $m_{\ell^{+} \ell^{-}}$distribution, peaking at the $Z$-boson mass, before applying the $m_{\ell^{+} \ell^{-}} \in[86.0,96.0] \mathrm{GeV}$ cut. Right: $m_{\text {recoil }}$ distribution, with the black curve peaking narrowly at $m_{h}$ on top of a continuum background, before requiring $m_{\text {recoil }} \in[123.0,127.0] \mathrm{GeV}$. At this stage of the selection the $t \bar{t}$ background is already negligible, and the distributions of the $2 \ell 2 \mathrm{~V}$ processes lie beyond the right edge of the plot. Some histograms in the right panel are scaled $(3 \times$ or $6 \times)$ after normalisation for visualisation purposes.

\section{3 $\quad Z B F$ channel event selection}

We now turn our attention to the $e^{+} e^{-} \rightarrow e^{+} e^{-} h Z B F$ mechanism, ${ }^{11}$ a $t$-channel process with a cross-section that grows with energy as $\sigma \sim \log ^{2}\left(s / m_{Z}^{2}\right)$. At the $\mathrm{ILC}_{250}$ this process interferes constructively with the $Z h$ channel, and the reduced phase-space makes it difficult to disentangle the kinematic features, such as a high-energy electron-positron pair in opposite regions of the detector, or $m_{e^{+} e^{-}}$well above $m_{Z}$. However, from figure 3 it can be seen that at $\sim 500 \mathrm{GeV}$ its cross-section is already larger than the $Z h$ one. At the same time, the latter is characterised by an on-shell $Z$-boson (see figure 4 ). The former features a highly energetic forward/backward electron-positron pair. So it provides

\footnotetext{
${ }^{11}$ On itself, this process seems not to be of much interest in the literature [79-81].
} 
access to observables involving the $Z$ and $h$ bosons at high-energy $e^{+} e^{-}$colliders, such as $\mathrm{ILC}_{1000}$ and $\mathrm{CLIC}_{3000}$, where beam polarisation also plays an important role in enhancing the cross-section of chirality-dependent processes.

A simplified cut-based analysis is performed on the stable final-state objects after running a detector simulation (see section 3.1) on the $Z B F$-like Monte Carlo samples at $\mathrm{ILC}_{250,1000}$ and $\mathrm{CLIC}_{3000}$. As before, we use $m_{e^{+} e^{-}}$along with $m_{\text {recoil }}$ in eq. (3.2) as the main background-discriminating variables, since $m_{\text {recoil }}$ displays the Higgs resonance as a sharp, narrow peak on top of a continuum background, and the broad $m_{e^{+} e^{-}}$distribution tends to favour large values well above $m_{Z}$ at $\mathrm{ILC}_{1000}$ and $\mathrm{CLIC}_{3000}$ energies, while for $\mathrm{ILC}_{250}$ there is large contamination from the $Z h$ process. As the centre-of-mass energy, $\sqrt{s}$, becomes larger, the final-state leptons tend to have larger values of $\left|y^{e}\right|$; however, a major experimental limitation is the absence of electromagnetic calorimeters available for electron tracking and reconstruction in the forward region of the detectors, reducing the phase-space (and in consequence, the cross-section) available for the study and characterisation of this process.

In practice, we impose a basic set of cuts, beginning with $y^{e^{+}} \cdot y^{e^{-}}<0$ to ensure that the electrons and positrons are in opposite regions of the detector, the fundamental feature of a forward process. Moreover, a large rapidity gap $\left|\Delta y_{e^{+}} e^{-}\right|$is expected, but not observed at the $\mathrm{ILC}_{250}$, although it is present at higher energies; at $\mathrm{ILC}_{1000}\left(\mathrm{CLIC}_{3000}\right)$ we demand $\left|\Delta y_{e^{+} e^{-}}\right| \in[3,5]([3.5,5])$. To further discriminate background events, we require $m_{e^{+} e^{-}} \in\{[105,130],[600,880],[2100,2880]\} \mathrm{GeV}$, and to control the effects of ISR, FSR, and beamstrahlung on the shape of the $m_{\text {recoil }}$ distribution, we impose the cut $p_{T}^{e^{+} e^{-}} \in$ $\{[20,50],[80,300],[150,800]\} \mathrm{GeV}$ at ILC $_{250,1000}$ and CLIC $_{3000}$, respectively. Finally, from eq. (3.2) and as done for the $Z h$ channel (see section 3.2), we apply a hard cut on the recoil system's mass $m_{\text {recoil }} \in[123,127] \mathrm{GeV}$ since there is a narrow peak at $m_{h} \sim 125 \mathrm{GeV}$, on top of a continuum background as expected. The $\left|\Delta y_{e^{+} e^{-}}\right|$and $m_{e^{+} e^{-}}$distributions for the $\mathrm{CLIC}_{3000}$ with right polarisation can be seen in figure 5, and the impact of the event selection on the cross-sections for the dominant SM processes under consideration is shown in table 5 for the three centre-of-mass energies, as well as for the left and right polarisations. Being a $t$-channel process, the $Z B F$ channel dominates over $Z h$ at high energies and, as such, the $\mathrm{ILC}_{250}$ is not a promising avenue to test the effects of the $\mathrm{D} 6$ operators under consideration. As for the $Z h$ scenario, we will only focus on $\mathrm{ILC}_{1000}$ and $\mathrm{CLIC}_{3000}$. As before, the kinematic distributions used to perform the event selection show similar shapes/behaviour in both polarisation settings.

\section{Projected sensitivities to EFT couplings}

In this section, we present the final sensitivity projections for the EFT couplings. We first generate samples with the EFT couplings turned on and apply the analysis strategy described in the previous section to obtain these. Then, for both the $Z h$ and $Z B F$ processes, we generate samples for enough points in the EFT parameter space so that it is possible to ascertain all the interference and EFT squared contributions as a function of the Wilson coefficients of the operators in table 2 . 

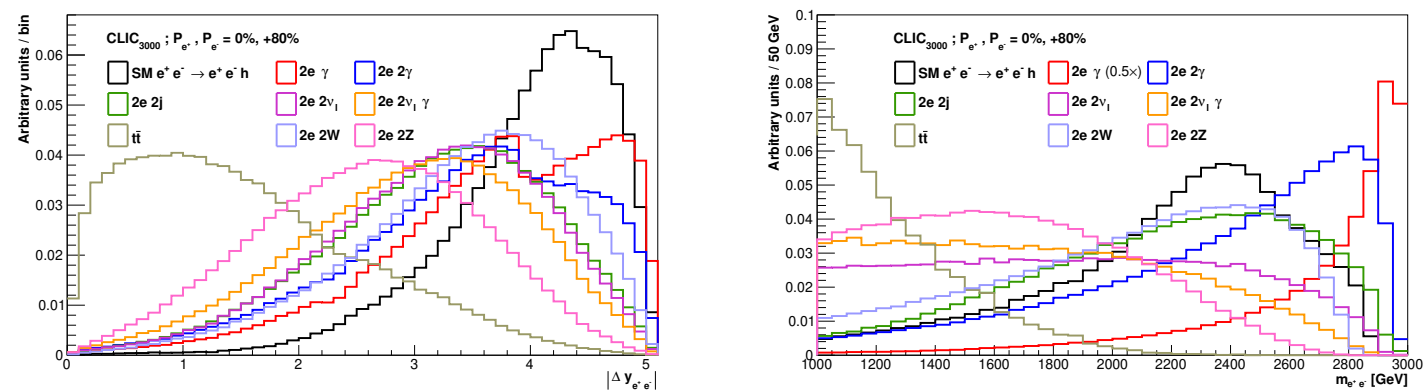

Figure 5. Some plots of the $\mathrm{CLIC}_{3000} Z B F$ selection with the right polarisation for the SM-driven $e^{+} e^{-} \rightarrow e^{+} e^{-} h$ (black), $2 e \gamma$ (red), $2 e 2 \gamma$ (blue), $2 e 2 j$ (green), $2 e 2 \nu_{\ell}$ (magenta), $2 e 2 \nu_{\ell} \gamma$ (orange), $t \bar{t}$ (brown), $2 e 2 W$ (lilac), and $2 e 2 Z$ (pink) processes. All histograms are normalised to unity. Left: $\left|\Delta y_{e^{+} e^{-}}\right|$distribution, showing the forward nature of the $e^{+} e^{-} \rightarrow e^{+} e^{-} h$ single-Higgs production process at high energies. Right: $m_{e^{+} e^{-}}$distribution before requiring $m_{e^{+} e^{-}} \in[2100.0,2880.0] \mathrm{GeV}$. The $2 e \gamma$ histogram in the right panel is scaled $(0.5 \times)$ after normalisation for visualisation purposes. Note that, although the $Z h$ and $Z B F$ diagrams interfere with each other, at high energies the $t$ channel $Z B F$ dominates over its $s$-channel counterpart $Z h$.

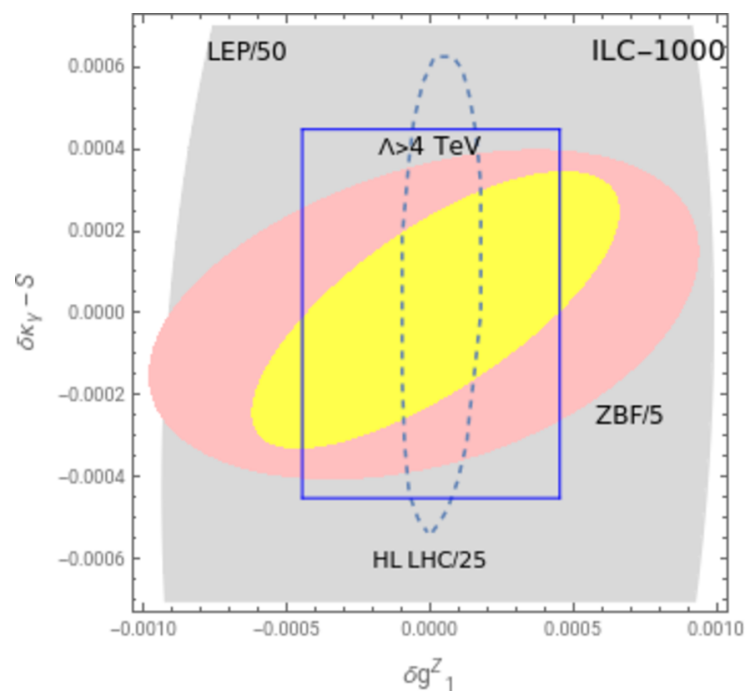

(a)

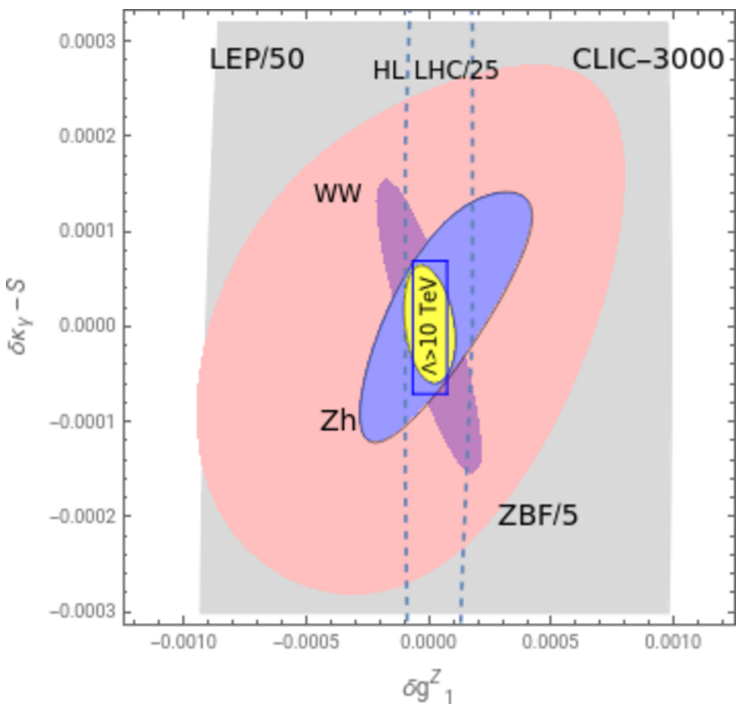

(b)

Figure 6. (a) Projected sensitivities for the case of universal new physics for $\operatorname{ILC}_{1000}$ and their comparison with LEP bounds [82] and HL-LHC projections [38] (b) Projected sensitivities for the case of universal new physics for CLIC $_{3000}$ and their comparison with LEP bounds and HL-LHC projections. We have assumed $W=Y=0$. See the text for more details. 


\begin{tabular}{|c|c|c|c|c|c|c|}
\hline$\sigma_{\sqrt{s}}^{\text {stage }}[\mathrm{fb}]$ & $\mathrm{SM}$ & $2 e \gamma$ & $2 e 2 \gamma$ & $2 e 2 \nu_{\ell}$ & $2 e 2 \nu_{\ell} \gamma$ & $2 e 2 j$ \\
\hline$\sigma_{250}^{\text {in }}$ & $0.88 / 0.66$ & $47354.7 / 46966$ & $628.53 / 620.28$ & $1348.33 / 99.46$ & $59.79 / 4.29$ & $125.27 / 115.97$ \\
$\sigma_{250}^{\text {out }}$ & $0.26 / 0.19$ & $<10^{-4}$ & $0.37 / 0.34$ & $1.57 / 0.13$ & $0.11 / 0.01$ & $0.033 / 0.024$ \\
\hline$\sigma_{1000}^{\text {in }}$ & $14.02 / 10.54$ & $9651.21 / 9221.23$ & $394.58 / 376.18$ & $430.13 / 59.66$ & $36.89 / 5.04$ & $93.29 / 76.42$ \\
$\sigma_{1000}^{\text {out }}$ & $2.52 / 1.92$ & $<10^{-4}$ & $0.034 / 0.030$ & $0.099 / 0.016$ & $0.0045 / 0.0017$ & $0.024 / 0.012$ \\
\hline$\sigma_{3000}^{\text {in }}$ & $4.11 / 3.08$ & $1754.91 / 1631$ & $115.66 / 107.46$ & $154.04 / 29.84$ & $17.45 / 3.56$ & $42.19 / 33.55$ \\
$\sigma_{3000}^{\text {out }}$ & $0.22 / 0.15$ & $<10^{-4}$ & $0.0052 / 0.0054$ & $0.0084 / 0.0022$ & $8.4 / 4.7\left(\times 10^{-4}\right)$ & $0.0033 / 0.0018$ \\
\hline
\end{tabular}

Table 5. Cross-sections, $\sigma$, in $\mathrm{fb}$, for the $Z B F$-like SM-driven signal $e^{+} e^{-} \rightarrow e^{+} e^{-} h$ and its dominant backgrounds before $($ in $)$ and after (out) event selection at $\sqrt{s}=\{250,1000,3000\} \mathrm{GeV}$. We consider the left and right beam polarisations $P_{e^{+}}, P_{e^{-}}$for each $\sqrt{s}$, reported as left/right (see text for details). For the $t \bar{t}, e^{+} e^{-} W^{+} W^{-}$, and $e^{+} e^{-} Z Z$ channels, $\sigma^{\text {in }}$ (in fb) $\sim 1.52 / 2.38$, $15.03 / 2.69$, and $0.04 / 0.02$, respectively at $1 \mathrm{TeV}$. At $3 \mathrm{TeV}$, the respective numbers are $0.12 / 0.21$, $29.84 / 3.88$, and $0.08 / 0.05$. For all cases, $\sigma^{\text {out }}<10^{-4} \mathrm{fb}$. For $\sqrt{s}=250 \mathrm{GeV}$, the kinematic phase space is not open for any of these three channels.

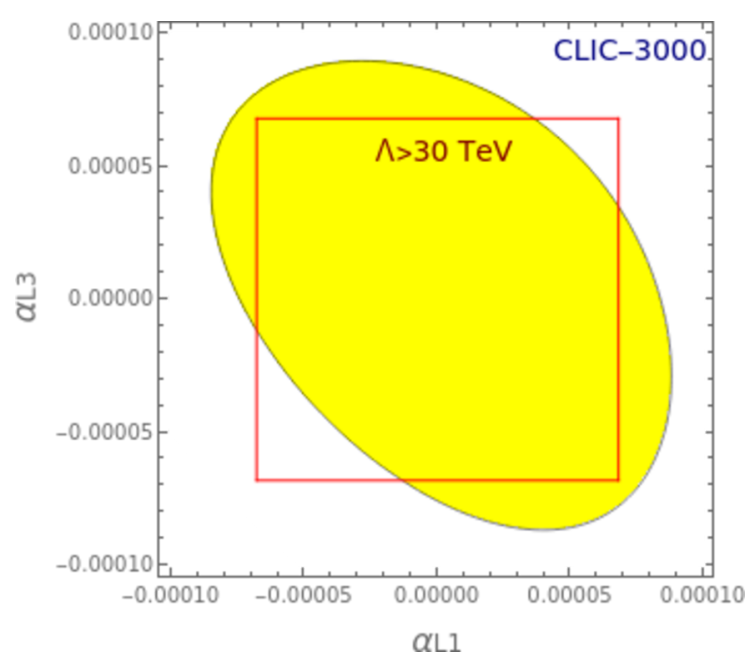

(a)

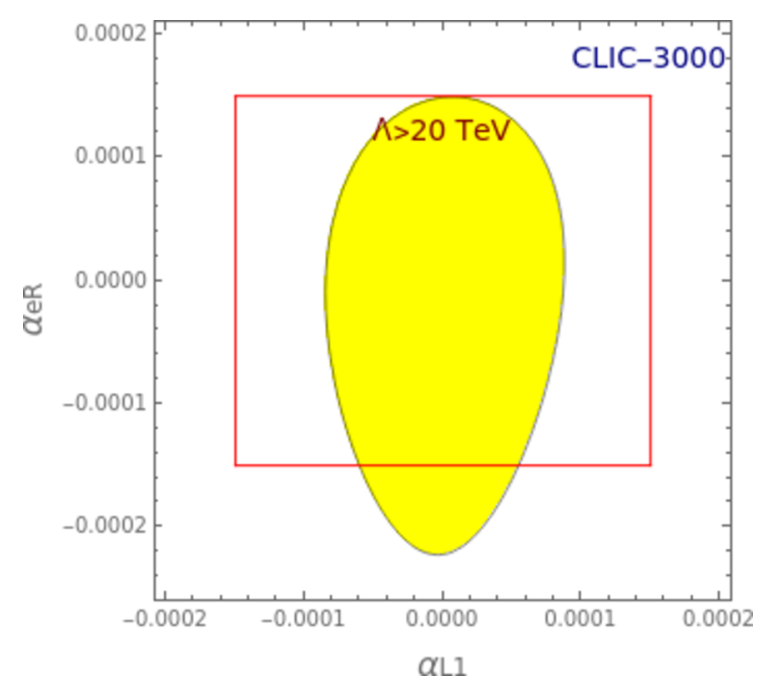

(b)

Figure 7. Projected sensitivities for the leptonic high-energy primaries, $\alpha_{e_{R}}=-c_{R}^{e} v^{2} / \Lambda^{2}, \alpha_{L 1}=$ $-c_{L}^{l,(1)} v^{2} / \Lambda^{2} \alpha_{L 3}=-c_{L}^{l,(3)} v^{2} / \Lambda^{2}$, at $\mathrm{CLIC}_{3000}$ in 2-dimensional planes where the third parameter has been marginalised over. See the text for more details.

In the following, we will include both the interference term between the SMEFT and SM amplitudes, and the SMEFT squared term. We have checked that, both for the ZBF and the $Z h$ processes, the squared term is between $1 \%$ and $10 \%$ of the interference term if we consider all possible polarisations and center of mass energies. This shows that dimension-eight (D8) effects, which are of the order of the squared term for $\mathcal{O}(1)$ Wilson coefficients, are indeed negligible. This can also be inferred from the fact that the final scales probed in this study are much larger than the centre-of-mass energy of the process (so that the ratio of D8 to D6 effects, $s / \Lambda^{2} \ll 1$ ). 


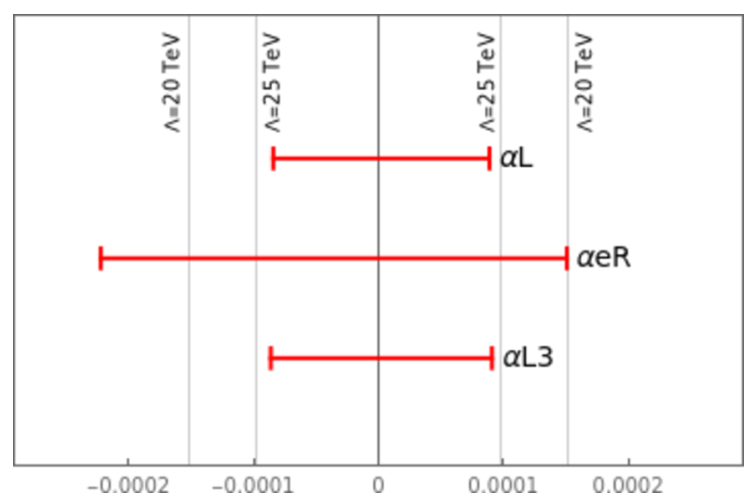

Figure 8. Projected sensitivities on the individual leptonic high-energy primaries, $\alpha_{e_{R}}=$ $-c_{R}^{e} v^{2} / \Lambda^{2}, \alpha_{L 1}=-c_{L}^{l,(1)} v^{2} / \Lambda^{2} \alpha_{L 3}=-c_{L}^{l,(3)} v^{2} / \Lambda^{2}$, at CLIC $_{3000}$ where the other two parameters have been marginalised over. See the text for more details.

First, let us consider the $Z h$ process. The experimental strategy used in section 3 to isolate the SM signal with respect to the other backgrounds is sufficient also to isolate the EFT signal. This is because, as discussed in section 2, the high-energy EFT contributions differ from the SM amplitudes only due to the lack of the $Z$-propagator, which implies that the EFT and SM contributions have the same angular dependence (see also ref. [37]). In any case, the dominant background for the EFT signal is by far the SM Higgs-strahlung process. To derive the projected sensitivity for the EFT couplings, we define a $\chi^{2}$ function as follows,

$$
\chi_{Z h}^{2}=\frac{\left(N_{Z h}^{\mathrm{exp}}-N_{Z h}^{\mathrm{obs}}\right)^{2}}{\sigma_{Z h}^{2}},
$$

where we have taken the SM to be our null hypothesis and $N_{Z h}^{\exp }$, denotes the expected number of events in the SM. The number of events observed, $N_{Z h}^{\text {obs }}$ will be assumed to be different from the SM due to the presence of EFT couplings. Finally, $\sigma_{i}$ quantifies the error including both the statistical and systematic uncertainties,

$$
\sigma_{Z h}=\sqrt{N_{Z h}^{\exp }+\left(\Delta_{\text {sys }} N_{Z h}^{\exp }\right)^{2}},
$$

where we take the percentage systematic uncertainty to be $\Delta_{\text {sys }}=0.03$ following ref. [51].

As far as the $Z B F$ process is concerned, once again, the experimental strategy required to isolate the SM $(Z B F)$ Higgs contribution from the other background processes in the previous section would also separate our signal, the EFT contribution. This is again because the dominant high-energy EFT contribution due to the operators in table 2 have an amplitude that is the same as the SM apart from a quadratic growth with respect to the Mandelstam variable $t$ (see also ref. [46]). We will use this quadratic growth with $t$ to distinguish the EFT contribution from the SM. To this end, we will use $p_{T}^{h}$, a variable highly correlated to $t$, as the discriminant. To obtain the projected sensitivity for the EFT couplings, we define the following $\chi^{2}$ function.

$$
\chi_{Z B F}^{2}=\sum_{i}^{N} \frac{\left(N_{i}^{\mathrm{exp}}-N_{i}^{\mathrm{obs}}\right)^{2}}{\sigma_{i}^{2}},
$$


where we again take the SM as our null hypothesis and, $N_{i}^{\exp }$, the expected number of events, is taken to be the SM value for the $i$ th bin in the $p_{T}^{h}$ distribution. The number of events observed in the $i$-th bin, $N_{i}^{\text {obs }}$ would be then assumed to be different from the SM due to the presence of EFT couplings. The total uncertainty for each bin, $\sigma_{i}$, is given by,

$$
\sigma_{i}=\sqrt{N_{i}^{\exp }+\left(\Delta_{\text {sys }} N_{i}^{\exp }\right)^{2}} .
$$

Finally, for the $e^{+} e^{-} \rightarrow W^{+} W^{-}$process, the $\chi^{2}$ function has been inferred from ref. [51] such that the bounds in table 15 of this paper are reproduced for both the semileptonic and fully hadronic cases. In order to statistically combine both these sub-processes, we then add the $\chi^{2}$ function for each case to obtain $\chi_{W W}^{2}$. To obtain our final bounds, we sum over the $\chi^{2}$ functions for all the different processes,

$$
\chi_{\text {total }}^{2}=\sum \chi_{Z h}^{2}+\sum \chi_{Z B F}^{2}+\sum \chi_{W W}^{2}
$$

where the summation in the terms above is over the different polarisation settings shown for $\sqrt{s}=1,3 \mathrm{TeV}$ in table 3 .

Combination of all channels for universal case. First, we derive the bounds in the scenario where new physics contributions can be encoded in universal operators of table 2. We can then present our bounds in a 2-dimensional plane by expressing the Wilson coefficients of the operators in the first column of table 2 as a function of 2 the universal parameters, $\left(\delta \kappa_{\gamma}-\hat{S}\right)$ and $\delta g_{1}^{Z}$, where we have ignored the $W$ and $Y$ contributions in eq. (2.3). The latter assumption is reasonable, first of all, because the $W$ and $Y$ parameters are expected to be constrained more strongly in Drell-Yan processes [51, 83, 84]. Secondly, there are some well-motivated UV examples where $W$ and $Y$ are small, as discussed in ref. [36].

We show the results for $\sqrt{s}=3 \mathrm{TeV}(\sqrt{s}=1 \mathrm{TeV})$ in figure 6 which includes the bounds from LEP [82] and projected sensitivities for HL-LHC from ref. [38]. It can be seen that in both cases, while the $Z B F$ produces very strong per-mile level bounds that far surpass the LEP bounds, it is still an order of magnitude smaller than the final bounds, including the process. This is because, while the total cross-section for the $Z B F$ process for both $\sqrt{s}=1 \mathrm{TeV}$ and $\sqrt{s}=3 \mathrm{TeV}$ is bigger than the Higgs-strahlung process, the number of events at high $p_{T}^{h}$ is much smaller; for instance the number of events becomes negligible for $p_{T}^{h}>300 \mathrm{GeV}\left(p_{T}^{h}>800 \mathrm{GeV}\right)$ in the $\sqrt{s}=1 \mathrm{TeV}(\sqrt{s}=3 \mathrm{TeV})$ case. In the $\sqrt{s}=1 \mathrm{TeV}$ case, the final bounds are indistinguishable from the bounds obtained just from the Higgs-strahlung process, which is much more sensitive than the $Z B F$ process. For the $\sqrt{s}=3 \mathrm{TeV}$ case, the bounds from the $e^{+} e^{-} \rightarrow W^{+} W^{-}$process turn out to be also very important as shown in figure 6 (b).

The LEP bounds are weaker by at least two orders of magnitudes with respect to our final bounds and thus have to be shrunk by a factor of 50 to be shown in the same plot. ${ }^{12}$ For the universal case, the processes considered here, and the corresponding ones for hadronic colliders with a $p p$ initial state, receive corrections from the same operators

\footnotetext{
${ }^{12}$ Note that the LEP bounds were derived assuming that the TGC couplings $\lambda_{\gamma}=\lambda_{Z}=0$. The bounds would be even weaker if these two couplings were not assumed to vanish but marginalised over.
} 
shown in the first column of table 2. This is what allows us to put bounds from HL-LHC, taken from ref. [38], in the same plane. Again our bounds are stronger by one order of magnitude than these HL-LHC projections.

In figure 6 we also show a region enclosed by a blue box to show the energy scale,

$$
\Lambda \sim \frac{m_{W}}{\sqrt{\delta g_{1}^{Z} c_{\theta_{W}}^{2}}}, \frac{m_{W}}{\sqrt{\delta \kappa_{\gamma}-\hat{S}}}
$$

that can be accessed in each case (see eq. (2.2) and eq. (2.3)). We see that scales as large as $10 \mathrm{TeV}(4 \mathrm{TeV})$ can be accessed in the $\sqrt{s}=3 \mathrm{TeV}(\sqrt{s}=1 \mathrm{TeV})$ case. This also shows that our study respects EFT validity considerations. The scales that can be probed here can be compared with the most powerful bounds from LEP on the $\hat{S}$-parameter which translates to the scales around $\Lambda \sim 1.6-2.5 \mathrm{TeV}[2]$.

Combination of all channels for general case. We now consider the general case where three linearly independent combinations of the leptonic high-energy primaries contribute to the processes considered in this work. ${ }^{13}$ As already discussed in section 2 , the three processes are sensitive to different combinations of the Wilson Coefficients of the operators in table 2. It is clear from eq. (2.1) and eq. (2.8) that the inclusion of the $W^{+} W^{-}$ processes now becomes crucial to leave no flat direction unconstrained because both the Higgs-strahlung and $Z B F$ processes are not sensitive to the EFT direction $\left(\alpha_{L 1}-\alpha_{L 3}\right)$. In figure 7 we provide the 2 dimensional bounds after marginalising over the third parameter. The individual $95 \%$ CL bounds on each of the three couplings after marginalising over the other two are as follows,

$$
\begin{aligned}
& \alpha_{L 1} \in[-8.5,8.8] \times 10^{-5} \\
& \alpha_{L 3} \in[-9,9] \times 10^{-4} \\
& \alpha_{e_{R}} \in[-2.2,1.5] \times 10^{-5}
\end{aligned}
$$

We show the same bounds graphically in figure 8 . In both figure 7 and figure 8 we provide information about the energy-scale, $\lambda \sim v / \sqrt{\alpha_{i}}$, that can be probed. We see that scales as high as 20-30 TeV can be probed.

The Wilson coefficients constrained in figure 7 and figure 8 can be expressed as a function of pseudo-observables already measured at LEP, as shown in eq. (2.7) which we rewrite here for convenience,

$$
\begin{aligned}
& \alpha_{L 1}=\frac{c_{\theta_{W}}}{g}\left(\delta g_{e_{L}}^{Z}+\delta g_{\nu_{L}}^{Z}\right)+s_{\theta_{W}}^{2} \delta g_{1}^{Z}-t_{\theta_{W}}^{2} \delta \kappa_{\gamma} \\
& \alpha_{L 3}=\frac{c_{\theta_{W}}}{g}\left(\delta g_{e_{L}}^{Z}-\delta g_{\nu_{L}}^{Z}\right)+c_{\theta_{W}}^{2} \delta g_{1}^{Z} \\
& \alpha_{e_{R}}=\frac{2 c_{\theta_{W}}}{g} \delta g_{e_{R}}^{Z}+2 s_{\theta_{W}}^{2} \delta g_{1}^{Z}-2 t_{\theta_{W}}^{2} \delta \kappa_{\gamma} .
\end{aligned}
$$

We can then use the above relations to compare our bounds with existing LEP bounds or projected HL-LHC sensitivities. The bounds on the right-hand side of the above relations

\footnotetext{
${ }^{13}$ Note that this does not imply that the three high-energy primaries are statistically independent parameters.
} 
arise mainly from the LEP (or projected HL-LHC) bounds on the TGCs, which are at least 100 (10) times weaker than the bounds in eq. (4.3). One can imagine a UV scenario where the EFT contribution to the TGCs vanishes and the bound on the right-hand side above arises from leptonic decays of the $Z$-boson, which were measured very precisely at LEP. These bounds are given by [12],

$$
\begin{aligned}
& \delta g_{e_{L}}^{Z} \in[-1,9] \times 10^{-4} \\
& \delta g_{e_{R}}^{Z} \in[-4,2] \times 10^{-4}
\end{aligned}
$$

We see that the bounds in eq. (4.3) are more powerful compared to even the LEP $Z$-pole bounds above.

\section{Conclusions}

Using the multi-dimensional space of SMEFT has now become the standard way to parametrise indirect effects at LHC and other future colliders [1-50]. Most SMEFT studies now aim to include all possible operators contributing to the list of considered processes. While such detailed and systematic studies are perhaps the best way to summarise the results of indirect searches comprehensively, one is often interested in a much simpler question: what is the highest scale that the collider can probe? It is sufficient to include only the most sensitive effects that dominate at high energies to answer this question. In this work, we answer this question in the context of electroweak processes at high-energy lepton colliders like the $\mathrm{ILC}_{1000}$ and $\mathrm{CLIC}_{3000}$. To this end, we identify the leading EFT effects that grow with energy and perform a 'high-energy fit' by including only the corresponding operators.

The processes we include are shown in table 1. Three linear combinations of the operators in table 2, the so-called leptonic high energy primaries, contribute to these processes at high-energies as shown in eq. (2.1), eq. (2.2), eq. (2.4) and eq. (2.8). These effects are larger than other EFT effects that do not grow with energy by a factor $s / m_{Z}^{2}$ which translates to two orders of magnitude for $\mathrm{ILC}_{1000}$ and $\mathrm{CLIC}_{3000} \cdot{ }^{14}$

Our final sensitivity estimates are shown in figure 6-8 and eq. (4.3). These estimates surpass existing LEP bounds by at least two orders of magnitude and projections for HLLHC by at least an order of magnitude. We have also shown in figure 6-8, the corresponding scales that these colliders can access. We see that the effects studied in this work can probe scales up to tens of $\mathrm{TeV}$, which corresponds to about $10^{-20}-10^{-19} \mathrm{~m}$. This would be the highest energy scale, and the smallest length scale, probed in Higgs/electroweak physics making these lepton colliders the ultimate microscopes to study fundamental physics.

\section{Acknowledgments}

We thank Keith R.K. Ellis, Rikkert Frederix, Valentin Hirschi, Shilpi Jain, Olivier Mattelaer, Rachel C. Rosten, and Zhuoni Qian for helpful exchanges. SB acknowledges the

\footnotetext{
${ }^{14}$ Even EFT effects that grow linearly with energy with respect to the leading SM piece will be smaller than these effects by one order of magnitude.
} 
support received from IPPP, Durham, U.K., where a part of this work was performed. SB is also grateful for the computing support received from IPPP, Durham. OOV acknowledges the grant received from the Mexican National Council for Science and Technology (CONACYT) - grant number - 460869 .

\section{A Contribution of operators to anomalous couplings}

In this appendix we connect the Wilson coefficients of the operators in table 2 to anomalous couplings for both the universal and general case. For the universal case, the relevant Lagragian is,

$$
\begin{aligned}
\Delta \mathcal{L}_{\text {univ }}= & -\frac{\hat{T}}{2} \frac{m_{Z}^{2}}{2} Z_{\mu} Z^{\mu}-\frac{\hat{S}}{4 m_{W}^{2}} \frac{g g^{\prime} v^{2}}{2}\left(W_{\mu \nu}^{3} B^{\mu \nu}\right)-\frac{W}{2 m_{W}^{2}}\left(\partial^{\mu} W_{\mu \nu}^{3}\right)^{2}-\frac{Y}{2 m_{W}^{2}}\left(\partial^{\mu} B_{\mu \nu}\right)^{2} \quad \text { (A.1) } \\
& +i g \delta g_{1}^{Z} c_{\theta_{W}} Z^{\mu}\left(W^{+\nu} W_{\mu \nu}^{-}-W^{-\nu} W_{\mu \nu}^{+}\right)+i g\left(\delta \kappa_{z} c_{\theta_{W}} Z^{\mu \nu}+\delta \kappa_{\gamma} s_{\theta_{W}} \hat{A}^{\mu \nu}\right) W_{\mu}^{+} W_{\nu}^{-},
\end{aligned}
$$

where at D6 level the following relationship holds, $\delta \kappa_{Z}=\delta g_{1}^{Z}-t_{\theta_{W}}^{2} \delta \kappa_{\gamma}$. Here $\hat{S}$ and $\hat{T}$ are the Peskin-Takeuchi parameters [85], $W$ and $Y$ are two-other Electroweak Precision observables defined in ref. [86] and the anomalous TGCs $\delta g_{1}^{Z}$ and $\delta \kappa_{\gamma}$ were first defined in ref. [87]. The contribution of the Wilson coefficients of the universal operators in the SILH basis is given by,

$$
\begin{aligned}
\hat{T} & =\frac{v^{2}}{\Lambda^{2}} c_{T}, \quad \hat{S}=\frac{m_{W}^{2}}{\Lambda^{2}}\left(c_{W}+c_{B}\right) & Y & =\frac{m_{W}^{2}}{\Lambda^{2}} c_{2 B} \quad W=\frac{m_{W}^{2}}{\Lambda^{2}} c_{2 W} \\
\delta g_{1}^{Z} & =-\frac{m_{W}^{2}}{\Lambda^{2}} \frac{1}{c_{\theta_{W}}^{2}}\left(c_{W}+c_{H W}\right) & \delta \kappa_{\gamma} & =-\frac{m_{W}^{2}}{\Lambda^{2}}\left(c_{H W}+c_{H B}\right),
\end{aligned}
$$

in the $\left\{\alpha_{e m}, G_{F}, m_{Z}\right\}$ scheme where $c_{T}$ is the Wilson coefficient of the operator $\mathcal{O}_{T}=$ $\left(H^{\dagger} \stackrel{\leftrightarrow}{D}_{\mu} H\right)^{2} / 2$. From the above equations we can derive the following relationships,

$$
\begin{aligned}
t_{\theta_{W}}^{2}\left(\delta \kappa_{\gamma}-\hat{S}-\delta g_{1}^{Z} c_{\theta_{W}}^{2}+Y\right) & =-\frac{m_{W}^{2}}{\Lambda^{2}}\left(c_{B}+c_{H B}-c_{2 B}\right) \\
\left(\delta g_{1}^{Z} c_{\theta_{W}}^{2}+W\right) & =-\frac{m_{W}^{2}}{\Lambda^{2}}\left(c_{W}+c_{H W}-c_{2 W}\right)
\end{aligned}
$$

which have been used in section 2 and also in section 4 to project our results into the plane in figure 6.

We can repeat the same exercise for the general case where, following ref. [14], we now use the Lagrangian,

$$
\begin{aligned}
\Delta \mathcal{L}_{6}= & \delta g_{L}^{W}\left(W_{\mu}^{+} \bar{\nu}_{L}^{e} \gamma^{\mu} e_{L}+h . c .\right)+g_{W L}^{h} \frac{h}{v}\left(W_{\mu}^{+} \bar{\nu}_{L} \gamma^{\mu} e_{L}+h . c .\right)+\sum_{l} \delta g_{l}^{Z} Z_{\mu} \bar{l} \gamma^{\mu} l+\sum_{l} g_{Z l}^{h} \frac{h}{v} Z_{\mu} \bar{l} \gamma^{\mu} l \\
& +i g \delta g_{1}^{Z} c_{\theta_{W}} Z^{\mu}\left(W^{+\nu} \hat{W}_{\mu \nu}^{-}-W^{-\nu} \hat{W}_{\mu \nu}^{+}\right)+i g\left(\delta \kappa_{z} c_{\theta_{W}} \hat{Z}^{\mu \nu}+\delta \kappa_{\gamma} s_{\theta_{W}} \hat{A}^{\mu \nu}\right) W_{\mu}^{+} W_{\nu}^{-} \text {(A.4) }
\end{aligned}
$$

where, for brevity, we have only included the first generation fermions, so that $l=e_{L}, e_{R}, \nu_{L}^{e}$ and $L$ is the first-generation lepton doublet. 
The operators of the Warsaw basis [3] in the right panel of table 2, give the following contributions to these vertices,

$$
\begin{aligned}
\delta g_{l}^{W}= & \frac{g}{\sqrt{2}} \frac{v^{2}}{\Lambda^{2}} c_{L}^{l,(3)}+\frac{\delta m_{Z}^{2}}{m_{Z}^{2}} \frac{\sqrt{2} g c_{\theta_{W}}^{2}}{4 s_{\theta_{W}}^{2}} \\
g_{W f}^{h}= & \sqrt{2} g \frac{v^{2}}{\Lambda^{2}} c_{L}^{l,(3)} \\
\delta g_{f}^{Z}= & -\frac{g Y_{f} s_{\theta_{W}}}{c_{\theta_{W}}^{2}} \frac{v^{2}}{\Lambda^{2}} c_{W B}-\frac{g}{c_{\theta_{W}}} \frac{v^{2}}{\Lambda^{2}}\left(\left|T_{3}^{f}\right| c_{L}^{l,(1)}-T_{3}^{f} c_{L}^{l,(3)}+\left(1 / 2-\left|T_{3}^{f}\right|\right) c_{R}^{e}\right) \\
& +\frac{\delta m_{Z}^{2}}{m_{Z}^{2}} \frac{g}{2 c_{\theta_{W}} s_{\theta_{W}}^{2}}\left(T_{3} c_{\theta_{W}}^{2}+Y_{f} s_{\theta_{W}}^{2}\right) \\
g_{Z f}^{h}= & -\frac{2 g}{c_{\theta_{W}}} \frac{v^{2}}{\Lambda^{2}}\left(\left|T_{3}^{f}\right| c_{L}^{l,(1)}-T_{3}^{f} c_{L}^{l,(3)}+\left(1 / 2-\left|T_{3}^{f}\right|\right) c_{R}^{e}\right) \\
\delta g_{1}^{Z}= & \frac{1}{2 s_{\theta_{W}}^{2}} \frac{\delta m_{Z}^{2}}{m_{Z}^{2}} \\
\delta \kappa_{\gamma}= & \frac{1}{t_{\theta_{W}}} \frac{v^{2}}{\Lambda^{2}} c_{W B}
\end{aligned}
$$

where we have now used $\left(m_{W}, m_{Z}, \alpha_{e m}\right)$ as our input parameters following ref. [14]. In the above equations, the term,

$$
\frac{\delta m_{Z}^{2}}{m_{Z}^{2}}=\frac{v^{2}}{\Lambda^{2}}\left(2 t_{\theta_{W}} c_{W B}+\frac{c_{H D}}{2}\right)
$$

corresponds to the shift in the input parameter, $m_{Z}$, due to the operators $\mathcal{O}_{W B}$ and $\mathcal{O}_{H D}$ defined in ref. [44]. Using the above equations, one can derive the following relationships,

$$
\begin{aligned}
c_{L}^{l,(1)} \frac{v^{2}}{\Lambda^{2}} & =-\frac{c_{\theta_{W}}}{g}\left(\delta g_{e_{L}}^{Z}+\delta g_{\nu_{L}}^{Z}\right)-s_{\theta_{W}}^{2} \delta g_{1}^{Z}+t_{\theta_{W}}^{2} \delta \kappa_{\gamma} \\
c_{L}^{l,(3)} \frac{v^{2}}{\Lambda^{2}} & =-\frac{c_{\theta_{W}}}{g}\left(\delta g_{e_{L}}^{Z}-\delta g_{\nu_{L}}^{Z}\right)-c_{\theta_{W}}^{2} \delta g_{1}^{Z} \\
c_{R}^{e} \frac{v^{2}}{\Lambda^{2}} & =-\frac{2 c_{\theta_{W}}}{g} \delta g_{e_{R}}^{Z}-2 s_{\theta_{W}}^{2} \delta g_{1}^{Z}+2 t_{\theta_{W}}^{2} \delta \kappa_{\gamma}
\end{aligned}
$$

that have been used in section 2 and section 4 .

Open Access. This article is distributed under the terms of the Creative Commons Attribution License (CC-BY 4.0), which permits any use, distribution and reproduction in any medium, provided the original author(s) and source are credited.

\section{References}

[1] W. Buchmüller and D. Wyler, Effective Lagrangian analysis of new interactions and flavor conservation, Nucl. Phys. B 268 (1986) 621 [INSPIRE].

[2] G.F. Giudice, C. Grojean, A. Pomarol and R. Rattazzi, The strongly-interacting light Higgs, JHEP 06 (2007) 045 [hep-ph/0703164] [INSPIRE]. 
[3] B. Grzadkowski, M. Iskrzynski, M. Misiak and J. Rosiek, Dimension-six terms in the Standard Model Lagrangian, JHEP 10 (2010) 085 [arXiv: 1008.4884] [INSPIRE].

[4] R.S. Gupta, Probing quartic neutral gauge boson couplings using diffractive photon fusion at the LHC, Phys. Rev. D 85 (2012) 014006 [arXiv:1111.3354] [InSPIRE].

[5] R.S. Gupta, H. Rzehak and J.D. Wells, How well do we need to measure Higgs boson couplings?, Phys. Rev. D 86 (2012) 095001 [arXiv:1206.3560] [INSPIRE].

[6] S. Banerjee, S. Mukhopadhyay and B. Mukhopadhyaya, New Higgs interactions and recent data from the LHC and the Tevatron, JHEP 10 (2012) 062 [arXiv:1207.3588] [INSPIRE].

[7] R.S. Gupta, M. Montull and F. Riva, SUSY faces its Higgs couplings, JHEP 04 (2013) 132 [arXiv: 1212.5240] [INSPIRE].

[8] S. Banerjee, S. Mukhopadhyay and B. Mukhopadhyaya, Higher dimensional operators and the LHC Higgs data: the role of modified kinematics, Phys. Rev. D 89 (2014) 053010 [arXiv: 1308.4860] [INSPIRE].

[9] R.S. Gupta, H. Rzehak and J.D. Wells, How well do we need to measure the Higgs boson mass and self-coupling?, Phys. Rev. D 88 (2013) 055024 [arXiv: 1305.6397] [INSPIRE].

[10] J. Elias-Miró, C. Grojean, R.S. Gupta and D. Marzocca, Scaling and tuning of EW and Higgs observables, JHEP 05 (2014) 019 [arXiv:1312.2928] [INSPIRE].

[11] R. Contino, M. Ghezzi, C. Grojean, M. Muhlleitner and M. Spira, Effective Lagrangian for a light Higgs-like scalar, JHEP 07 (2013) 035 [arXiv:1303.3876] [INSPIRE].

[12] A. Falkowski and F. Riva, Model-independent precision constraints on dimension-6 operators, JHEP 02 (2015) 039 [arXiv: 1411.0669] [INSPIRE].

[13] C. Englert and M. Spannowsky, Effective theories and measurements at colliders, Phys. Lett. $B \mathbf{7 4 0}$ (2015) 8 [arXiv:1408.5147] [INSPIRE].

[14] R.S. Gupta, A. Pomarol and F. Riva, BSM primary effects, Phys. Rev. D 91 (2015) 035001 [arXiv: 1405.0181] [INSPIRE].

[15] G. Amar et al., Exploration of the tensor structure of the Higgs boson coupling to weak bosons in $e^{+} e^{-}$collisions, JHEP 02 (2015) 128 [arXiv:1405.3957] [INSPIRE].

[16] M. Buschmann, D. Goncalves, S. Kuttimalai, M. Schonherr, F. Krauss and T. Plehn, Mass effects in the Higgs-gluon coupling: boosted vs off-shell production, JHEP 02 (2015) 038 [arXiv: 1410.5806] [INSPIRE].

[17] N. Craig, M. Farina, M. McCullough and M. Perelstein, Precision higgsstrahlung as a probe of new physics, JHEP 03 (2015) 146 [arXiv:1411.0676] [INSPIRE].

[18] J. Ellis, V. Sanz and T. You, Complete Higgs sector constraints on dimension-6 operators, JHEP 07 (2014) 036 [arXiv: 1404.3667] [INSPIRE].

[19] J. Ellis, V. Sanz and T. You, The effective Standard Model after LHC run I, JHEP 03 (2015) 157 [arXiv: 1410.7703] [inSPIRE].

[20] S. Banerjee, T. Mandal, B. Mellado and B. Mukhopadhyaya, Cornering dimension-6 HVV interactions at high luminosity LHC: the role of event ratios, JHEP 09 (2015) 057 [arXiv: 1505.00226] [INSPIRE].

[21] C. Englert, R. Kogler, H. Schulz and M. Spannowsky, Higgs coupling measurements at the LHC, Eur. Phys. J. C 76 (2016) 393 [arXiv:1511.05170] [InSPIRE]. 
[22] D. Ghosh, R.S. Gupta and G. Perez, Is the Higgs mechanism of fermion mass generation a fact? A Yukawa-less first-two-generation model, Phys. Lett. B 755 (2016) 504 [arXiv: 1508.01501] [INSPIRE].

[23] C. Degrande, B. Fuks, K. Mawatari, K. Mimasu and V. Sanz, Electroweak Higgs boson production in the Standard Model effective field theory beyond leading order in QCD, Eur. Phys. J. C 77 (2017) 262 [arXiv:1609.04833] [InSPIRE].

[24] J. Cohen, S. Bar-Shalom and G. Eilam, Contact interactions in Higgs-vector boson associated production at the ILC, Phys. Rev. D 94 (2016) 035030 [arXiv:1602.01698] [InSPIRE].

[25] S.-F. Ge, H.-J. He and R.-Q. Xiao, Probing new physics scales from Higgs and electroweak observables at $e^{+} e^{-}$Higgs factory, JHEP 10 (2016) 007 [arXiv: 1603.03385] [INSPIRE].

[26] R. Contino, A. Falkowski, F. Goertz, C. Grojean and F. Riva, On the validity of the effective field theory approach to SM precision tests, JHEP 07 (2016) 144 [arXiv: 1604.06444] [INSPIRE].

[27] A. Biekötter, J. Brehmer and T. Plehn, Extending the limits of Higgs effective theory, Phys. Rev. D 94 (2016) 055032 [arXiv: 1602.05202] [INSPIRE].

[28] J. de Blas et al., Electroweak precision observables and Higgs-boson signal strengths in the Standard Model and beyond: present and future, JHEP 12 (2016) 135 [arXiv:1608.01509] [INSPIRE].

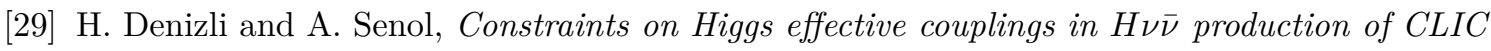
at $380 \mathrm{GeV}$, Adv. High Energy Phys. 2018 (2018) 1627051 [arXiv:1707.03890] [INSPIRE].

[30] T. Barklow et al., Improved formalism for precision Higgs coupling fits, Phys. Rev. D 97 (2018) 053003 [arXiv: 1708.08912] [INSPIRE].

[31] I. Brivio and M. Trott, The Standard Model as an effective field theory, Phys. Rept. $\mathbf{7 9 3}$ (2019) 1 [arXiv: 1706. 08945] [INSPIRE].

[32] T. Barklow, K. Fujii, S. Jung, M.E. Peskin and J. Tian, Model-independent determination of the triple Higgs coupling at $e^{+} e^{-}$colliders, Phys. Rev. D 97 (2018) 053004 [arXiv: 1708.09079] [INSPIRE].

[33] H. Khanpour and M. Mohammadi Najafabadi, Constraining Higgs boson effective couplings at electron-positron colliders, Phys. Rev. D 95 (2017) 055026 [arXiv:1702.00951] [INSPIRE].

[34] C. Englert, R. Kogler, H. Schulz and M. Spannowsky, Higgs characterisation in the presence of theoretical uncertainties and invisible decays, Eur. Phys. J. C 77 (2017) 789 [arXiv: 1708.06355] [INSPIRE].

[35] G. Panico, F. Riva and A. Wulzer, Diboson interference resurrection, Phys. Lett. B 776 (2018) 473 [arXiv : 1708.07823] [INSPIRE].

[36] R. Franceschini, G. Panico, A. Pomarol, F. Riva and A. Wulzer, Electroweak precision tests in high-energy diboson processes, JHEP 02 (2018) 111 [arXiv:1712.01310] [INSPIRE].

[37] S. Banerjee, C. Englert, R.S. Gupta and M. Spannowsky, Probing electroweak precision physics via boosted Higgs-strahlung at the LHC, Phys. Rev. D 98 (2018) 095012 [arXiv: 1807.01796] [INSPIRE].

[38] C. Grojean, M. Montull and M. Riembau, Diboson at the LHC vs LEP, JHEP 03 (2019) 020 [arXiv: 1810.05149] [INSPIRE]. 
[39] A. Biekoetter, T. Corbett and T. Plehn, The gauge-Higgs legacy of the LHC run II, SciPost Phys. 6 (2019) 064 [arXiv:1812.07587] [INSPIRE].

[40] D. Goncalves and J. Nakamura, Boosting the $H \rightarrow$ invisibles searches with $Z$ boson polarization, Phys. Rev. D 99 (2019) 055021 [arXiv: 1809.07327] [INSPIRE].

[41] R. Gomez-Ambrosio, Studies of dimension-six EFT effects in vector boson scattering, Eur. Phys. J. C $\mathbf{7 9}$ (2019) 389 [arXiv:1809.04189] [InSPIRE].

[42] F.F. Freitas, C.K. Khosa and V. Sanz, Exploring the Standard Model EFT in VH production with machine learning, Phys. Rev. D 100 (2019) 035040 [arXiv: 1902.05803] [INSPIRE].

[43] S. Banerjee, R.S. Gupta, J.Y. Reiness and M. Spannowsky, Resolving the tensor structure of the Higgs coupling to Z-bosons via Higgs-strahlung, Phys. Rev. D 100 (2019) 115004 [arXiv: 1905.02728] [INSPIRE].

[44] S. Banerjee, R.S. Gupta, J.Y. Reiness, S. Seth and M. Spannowsky, Towards the ultimate differential SMEFT analysis, JHEP 09 (2020) 170 [arXiv: 1912.07628] [INSPIRE].

[45] A. Biekötter, R. Gomez-Ambrosio, P. Gregg, F. Krauss and M. Schönherr, Constraining

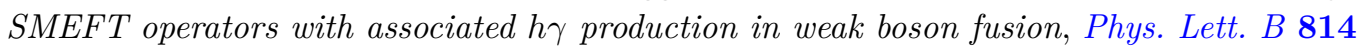
(2021) 136079 [arXiv:2003.06379] [INSPIRE].

[46] J.Y. Araz, S. Banerjee, R.S. Gupta and M. Spannowsky, Precision SMEFT bounds from the VBF Higgs at high transverse momentum, JHEP 04 (2021) 125 [arXiv:2011.03555] [INSPIRE].

[47] J. Ellis, M. Madigan, K. Mimasu, V. Sanz and T. You, Top, Higgs, diboson and electroweak fit to the Standard Model effective field theory, JHEP 04 (2021) 279 [arXiv:2012.02779] [INSPIRE].

[48] S. Banerjee, R.S. Gupta, O. Ochoa-Valeriano, M. Spannowsky and E. Venturini, A fully differential SMEFT analysis of the golden channel using the method of moments, JHEP 06 (2021) 031 [arXiv : 2012.11631] [INSPIRE].

[49] E.d.S. Almeida, A. Alves, O.J.P. Éboli and M.C. Gonzalez-Garcia, Electroweak legacy of the LHC run II, Phys. Rev. D 105 (2022) 013006 [arXiv:2108.04828] [INSPIRE].

[50] S. Chatterjee, N. Frohner, L. Lechner, R. Schöfbeck and D. Schwarz, Tree boosting for learning EFT parameters, arXiv:2107.10859 [INSPIRE].

[51] J. de Blas et al., The CLIC potential for new physics, arXiv:1812.02093 [INSPIRE].

[52] D. Buttazzo, D. Redigolo, F. Sala and A. Tesi, Fusing vectors into scalars at high energy lepton colliders, JHEP 11 (2018) 144 [arXiv: 1807.04743] [INSPIRE].

[53] D. Buttazzo, R. Franceschini and A. Wulzer, Two paths towards precision at a very high energy lepton collider, JHEP 05 (2021) 219 [arXiv:2012.11555] [INSPIRE].

[54] M.S. Chanowitz and M.K. Gaillard, The TeV physics of strongly interacting $W$ 's and $Z$ 's, Nucl. Phys. B 261 (1985) 379 [inSPIRE].

[55] A. Wulzer, An equivalent gauge and the equivalence theorem, Nucl. Phys. B $\mathbf{8 8 5}$ (2014) 97 [arXiv: 1309.6055] [INSPIRE].

[56] H. Yamamoto, Physics at International Linear Collider (ILC), J. Phys. Soc. Jap. 76 (2007) 111014 [arXiv: 0709.0899] [INSPIRE].

[57] H. Abramowicz et al., Higgs physics at the CLIC electron-positron linear collider, Eur. Phys. J. C 77 (2017) 475 [arXiv: 1608.07538] [INSPIRE]. 
[58] CLICDP, ILD CONCEPT Group collaboration, On the physics potential of ILC and CLIC, PoS CORFU2019 (2020) 037 [arXiv: 2004.14628] [INSPIRE].

[59] C. Degrande, C. Duhr, B. Fuks, D. Grellscheid, O. Mattelaer and T. Reiter, UFO - the Universal FeynRules Output, Comput. Phys. Commun. 183 (2012) 1201 [arXiv:1108.2040] [INSPIRE].

[60] A. Alloul, N.D. Christensen, C. Degrande, C. Duhr and B. Fuks, FeynRules $2.0-a$ complete toolbox for tree-level phenomenology, Comput. Phys. Commun. 185 (2014) 2250 [arXiv: 1310.1921] [INSPIRE].

[61] T. Behnke et al. eds., The International Linear Collider technical design report - volume 1: executive summary, arXiv:1306.6327 [INSPIRE].

[62] D.M. Asner et al., ILC Higgs white paper, in Community Summer study 2013: Snowmass on the Mississippi, (2013) [arXiv:1310.0763] [INSPIRE].

[63] L. Linssen, A. Miyamoto, M. Stanitzki and H. Weerts, eds., Physics and detectors at CLIC: CLIC conceptual design report, CERN Yellow Repts., CERN, Geneva, Switzerland (2012) [arXiv: 1202.5940] [INSPIRE].

[64] J. de Blas et al., Higgs boson studies at future particle colliders, JHEP 01 (2020) 139 [arXiv: 1905.03764] [INSPIRE].

[65] G. Moortgat-Pick et al., The role of polarized positrons and electrons in revealing fundamental interactions at the linear collider, Phys. Rept. 460 (2008) 131 [hep-ph/0507011] [INSPIRE].

[66] G. Durieux, C. Grojean, J. Gu and K. Wang, The leptonic future of the Higgs, JHEP 09 (2017) 014 [arXiv : 1704. 02333] [INSPIRE].

[67] K. Fujii et al., The role of positron polarization for the inital $250 \mathrm{GeV}$ stage of the International Linear Collider, arXiv:1801.02840 [INSPIRE].

[68] Linear Collider collaboration, Polarised beams at future $e^{+} e^{-}$colliders, PoS ICHEP2020 (2021) 691 [arXiv: 2012 .11267] [INSPIRE].

[69] J. Alwall et al., The automated computation of tree-level and next-to-leading order differential cross sections, and their matching to parton shower simulations, JHEP 07 (2014) 079 [arXiv: 1405.0301] [INSPIRE].

[70] T. Sjöstrand et al., An introduction to PYTHIA 8.2, Comput. Phys. Commun. 191 (2015) 159 [arXiv: 1410.3012] [INSPIRE].

[71] DELPHES 3 collaboration, DELPHES 3, a modular framework for fast simulation of a generic collider experiment, JHEP 02 (2014) 057 [arXiv: 1307.6346] [INSPIRE].

[72] H. Abramowicz et al., The International Linear Collider technical design report - volume 4: detectors, arXiv:1306.6329 [INSPIRE].

[73] P. Demin and M. Selvaggi, Delphes Card ILD, https://github.com/delphes/delphes/blob/master/cards/delphes_card_ILD.tcl, accessed 1 June 2021.

[74] CLICDP collaboration, A detector for CLIC: main parameters and performance, Tech. Rep. CLICdp-Note-2018-005, CERN, Geneva, Switzerland (2018) [arXiv:1812.07337] [INSPIRE]. 
[75] E. Leogrande, P. Roloff, U. Schnoor and M. Weber, A DELPHES card for the CLIC detector, Tech. Rep. CLICdp-Note-2018-007, CERN, Geneva, Switzerland (2018) [arXiv: 1909.12728] [INSPIRE].

[76] LHC Higgs Cross Section Working Group collaboration, Handbook of LHC Higgs cross sections: 4. Deciphering the nature of the Higgs sector, arXiv:1610.07922 [INSPIRE].

[77] N. Craig, J. Gu, Z. Liu and K. Wang, Beyond Higgs couplings: probing the Higgs with angular observables at future $e^{+} e^{-}$colliders, JHEP 03 (2016) 050 [arXiv:1512.06877] [INSPIRE].

[78] J. Yan et al., Measurement of the Higgs boson mass and $e^{+} e^{-} \rightarrow Z H$ cross section using $Z \rightarrow \mu^{+} \mu^{-}$and $Z \rightarrow e^{+} e^{-}$at the ILC, Phys. Rev. D 94 (2016) 113002 [Erratum ibid. 103 (2021) 099903] [arXiv: 1604.07524] [INSPIRE].

[79] J.F. Gunion, T. Han and R. Sobey, Measuring the coupling of a Higgs boson to ZZ at linear colliders, Phys. Lett. B 429 (1998) 79 [hep-ph/9801317] [INSPIRE].

[80] T. Han and J. Jiang, CP violating ZZH coupling at $e^{+} e^{-}$linear colliders, Phys. Rev. D 63 (2001) 096007 [hep-ph/0011271] [INSPIRE].

[81] T. Han, Z. Liu, Z. Qian and J. Sayre, Improving Higgs coupling measurements through ZZ fusion at the ILC, Phys. Rev. D 91 (2015) 113007 [arXiv:1504.01399] [InSPIRE].

[82] ALEPH, DELPHI, L3, OPAL and LEP TGC Working Group collaborations, $A$ combination of preliminary results on gauge boson couplings measured by the LEP experiments, Tech. Rep. LEPEWWG-TGC-2003-01, CERN, Geneva, Switzerland (2003).

[83] R. Torre, L. Ricci and A. Wulzer, On the $W \& Y$ interpretation of high-energy Drell-Yan measurements, JHEP 02 (2021) 144 [arXiv:2008.12978] [INSPIRE].

[84] G. Panico, L. Ricci and A. Wulzer, High-energy EFT probes with fully differential Drell-Yan measurements, JHEP 07 (2021) 086 [arXiv:2103.10532] [INSPIRE].

[85] M.E. Peskin and T. Takeuchi, Estimation of oblique electroweak corrections, Phys. Rev. D 46 (1992) 381 [INSPIRE].

[86] R. Barbieri, A. Pomarol, R. Rattazzi and A. Strumia, Electroweak symmetry breaking after LEP-1 and LEP-2, Nucl. Phys. B 703 (2004) 127 [hep-ph/0405040] [InSPIRE].

[87] K. Hagiwara, R.D. Peccei, D. Zeppenfeld and K. Hikasa, Probing the weak boson sector in $e^{+} e^{-} \rightarrow W^{+} W^{-}$, Nucl. Phys. B 282 (1987) 253 [inSPIRE]. 